Contemporary Mathematics

\title{
Topological Hochschild homology and the Hasse-Weil zeta function
}

\author{
Lars Hesselholt
}

\section{Introduction}

In this paper, we consider the Tate cohomology of the circle group acting on the topological Hochschild homology of schemes. We show that in the case of a scheme smooth and proper over a finite field, this cohomology theory naturally gives rise to the cohomological interpretation of the Hasse-Weil zeta function by regularized determinants envisioned by Deninger $\mathbf{1 5}$. In this case, the periodicity of the zeta function is reflected by the periodicity of said cohomology theory, whereas neither is periodic in general.

Let $X$ be a scheme. Connes' periodic cyclic homology of $X$ may be defined to be the anticommutative graded ring $\operatorname{HP}_{*}(X)$ given by the Tate cohomology groups

$$
\operatorname{HP}_{i}(X)=\hat{H}^{-i}(\mathbb{T}, \mathrm{HH}(X))
$$

in the sense of Greenlees $[\mathbf{2 0}, \mathbf{3 2}$ of the circle group $\mathbb{T}$ acting on the Hochschild spectrum $\mathrm{HH}(X)$. By analogy, we consider the anticommutative graded ring $\mathrm{TP}_{*}(X)$ given by the Tate cohomology groups

$$
\operatorname{TP}_{i}(X)=\hat{H}^{-i}(\mathbb{T}, \operatorname{THH}(X))
$$

of $\mathbb{T}$ acting on Bökstedt's topological Hochschild spectrum $\operatorname{THH}(X)$ [7, 23. The latter is an implementation of Waldhausen's philosophy to replace the initial ring of algebra $\mathbb{Z}$ by the initial ring of higher algebra $\mathbb{S}$. The graded ring $\operatorname{TP}_{*}(X)$ is the abutment of the Tate spectral sequence

$$
E_{i, j}^{2}=H^{-i}\left(\mathbb{P}_{-\infty}^{\infty}(\mathbb{C}), \mathrm{THH}_{j}(X)\right) \Rightarrow \mathrm{TP}_{i+j}(X),
$$

which is multiplicative and converges conditionally in the sense of Boardman [6. The $E^{2}$-term is periodic with periodicity operator given by multiplication by

$$
t=c_{1}(\mathcal{O}(1)) \in H^{2}\left(\mathbb{P}^{\infty}(\mathbb{C}), \mathbb{Z}\right)=H^{2}\left(\mathbb{P}_{-\infty}^{\infty}(\mathbb{C}), \mathbb{Z}\right) .
$$

In Connes' theory, the periodicity element $t$ is an infinite cycle and is represented by a unique homotopy class $v \in \mathrm{HP}_{-2}(\mathbb{Z})$, the multiplication by which is Connes' $S$-operator. But in the topologically refined situation, the element $t$ does not in general survive the spectral sequence, and, if it does, is typically not represented

2010 Mathematics Subject Classification. Primary 11S40, 19D55; Secondary 14 F30.

Assistance from DNRF Niels Bohr Professorship is gratefully acknowledged.

(C) 0000 (copyright holder) 
by a unique homotopy class. In particular, the graded ring $\mathrm{TP}_{*}(X)$ is generally not periodic. However, by contrast to Connes' theory, the graded ring $\operatorname{TP}_{*}(X)$ is, in favorable situations, equipped with meromorphic Frobenius operators

$$
\mathrm{TP}_{*}(X) \stackrel{\varphi_{p}}{\longrightarrow} \mathrm{TP}_{*}(X)
$$

The operator $\varphi_{p}$ is obtained from the cyclotomic structure of $\mathrm{THH}(X)$ by a process that bears some resemblance to analytic continuation. In particular, it does not always exist, and, when it does, its construction requires some work.

Suppose now that $X$ is defined over a finite field $k$ of order $q=p^{r}$. In this situation, the periodicity element $t$ is an infinite cycle and is represented by a preferred homotopy class $v \in \mathrm{TP}_{-2}(k)$, the definition of which is given in Section 4 below. Moreover, there is a canonical identification of the ring $\mathrm{TP}_{0}(k)$ with the ring $W$ of $p$-typical Witt vectors in $k$. It follows that $\operatorname{TP}_{*}(X)$ is a 2-periodic anticommutative graded $W$-algebra. In this situation, the Frobenius operator $\varphi_{p}$ is defined after inverting $p$. Moreover, the $W$-linear operator $\varphi_{p}^{r}$ and the weight filtration of $\mathrm{TP}_{*}(X)$ determines the action of the geometric Frobenius $\operatorname{Fr}_{q}: X \rightarrow X$ on $\mathrm{TP}_{*}(X) \otimes_{W, \iota} \mathbb{C}$.

TheOREM A. Let $k$ be a finite field of order $q=p^{r}$, let $W$ be its ring of p-typical Witt vectors, and let $\iota: W \rightarrow \mathbb{C}$ be a choice of embedding. If $f: X \rightarrow \operatorname{Spec}(k)$ is a smooth and proper morphism of schemes, then, as meromorphic functions on $\mathbb{C}$,

$$
\zeta(X, s)=\frac{\operatorname{det}_{\infty}\left(s \cdot \mathrm{id}-\Theta \mid \mathrm{TP}_{\mathrm{od}}(X) \otimes_{W, \iota} \mathbb{C}\right)}{\operatorname{det}_{\infty}\left(s \cdot \mathrm{id}-\Theta \mid \mathrm{TP}_{\mathrm{ev}}(X) \otimes_{W, \iota} \mathbb{C}\right)},
$$

where $\Theta$ is a $\mathbb{C}$-linear graded derivation such that $q^{\Theta}=\operatorname{Fr}_{q}^{*}$ and $\Theta(v)=\frac{2 \pi i}{\log q} \cdot v$.

The graded derivation $\Theta$ is determined by the specified value on the periodicity class $v$ and by its values on $\mathrm{TP}_{0}(X) \otimes_{W, \iota} \mathbb{C}$ and $\mathrm{TP}_{1}(X) \otimes_{W, \iota} \mathbb{C}$, which are both finite dimensional $\mathbb{C}$-vector spaces. To specify the latter, we follow Deninger [15, Section 2.12] and decompose the Frobenius $\operatorname{Fr}_{q}^{*}=\operatorname{Fr}_{q, s}^{*} \circ \operatorname{Fr}_{q, u}^{*}$ in its semisimple and unipotent parts and define

$$
\Theta=\log _{q}\left(\operatorname{Fr}_{q}^{*}\right)=\log _{q}\left(\operatorname{Fr}_{q, s}^{*}\right)+\log _{q}\left(\operatorname{Fr}_{q, u}^{*}\right) .
$$

Here we use some choice of principal branch of the logarithm on the semisimple part and the series $\frac{1}{\log q} \log (-)$ on the unipotent part. Since, by naturality, $\operatorname{Fr}_{q}^{*}(v)=v$, the operator $\Theta$ may be seen as the most general solution to $q^{\Theta}=\operatorname{Fr}_{q}^{*}$, which is in accordance with Deninger's philosophy that $\Theta$ should be the infinitesimal generator of a Frobenius flow $\mathrm{Fr}_{t}^{*}$. We will recall the notions of regularized determinant and anomalous dimension in the final section of the paper.

The operator $s \cdot$ id $-\Theta$ has anomalous dimension zero. Therefore, we may fix any choice of a positive real scaling factor $\delta$ and state Theorem $\mathrm{A}$ as

$$
\zeta(X, s)=\frac{\operatorname{det}_{\infty}\left(\delta(s \cdot \operatorname{id}-\Theta) \mid \operatorname{TP}_{\mathrm{od}}(X) \otimes_{W, \iota} \mathbb{C}\right)}{\operatorname{det}_{\infty}\left(\delta(s \cdot \operatorname{id}-\Theta) \mid \mathrm{TP}_{\mathrm{ev}}(X) \otimes_{W, \iota} \mathbb{C}\right)}
$$

In particular, we may take $\delta=\frac{1}{2 \pi}$ as in the archimedean case [14, Theorem 1.1], where the scaling factor is essential.

We outline the proof of Theorem $\mathrm{A}$. The present proofl] uses the cohomological interpretation of the Hasse-Weil zeta function by crystalline cohomology established

\footnotetext{
${ }^{1}$ The recent proof of the Künneth formula for TP by Blumberg-Mandell [4, Theorem A] has facilitated a direct proof given by Tabuada [35 Theorem 3.27].
} 
by Berthelot in his thesis [2, Théorème VII.3.2.3],

$$
\zeta(X, s)=\frac{\operatorname{det}\left(\mathrm{id}-q^{-s} \operatorname{Fr}_{q}^{*} \mid H_{\mathrm{crys}}^{\mathrm{od}}(X / W) \otimes_{W, \iota} \mathbb{C}\right)}{\operatorname{det}\left(\mathrm{id}-q^{-s} \operatorname{Fr}_{q}^{*} \mid H_{\mathrm{crys}}^{\mathrm{ev}}(X / W) \otimes_{W, \iota} \mathbb{C}\right)} .
$$

The comparison theorem of Bloch-Deligne-Illusie [29. Théorème II.1.4] shows that, in this formula, the crystalline cohomology $H_{\text {crys }}^{i}(X / W)$ may be replaced by the hypercohomology $H^{i}\left(X, W \Omega_{X}{ }^{\prime}\right)$ with coefficients in the de Rham-Witt complex. Therefore, we have the two spectral sequences of hypercohomology, one of which gives rise to a natural spectral sequence of finite dimensional $\mathbb{C}$-vector spaces

$$
E_{2}^{i, j}=\left(\lim _{n, F} H^{i}\left(X, W_{n} \Omega_{X}^{j}\right)\right) \otimes_{W, \iota} \mathbb{C} \Rightarrow H_{\text {crys }}^{i+j}(X / W) \otimes_{W, \iota} \mathbb{C}
$$

called the conjugate spectral sequence. Here, the identification of the $E_{2}$-term also uses the higher Cartier isomorphism of Illusie-Raynaud [30, Proposition III.1.4]. We prove in Theorem 6.8 below that, as a consequence of the definition of $\mathrm{THH}(X)$ in [5, 17] and the calculation of the equivariant homotopy groups of $\operatorname{THH}\left(\mathcal{O}_{X}\right)$ in [21, Theorem $\mathrm{B}$ ], the same finite dimensional $\mathbb{C}$-vector spaces appear in the $E^{2}$-term of a natural spectral sequence

$$
E_{i, j}^{2}=\bigoplus_{m \in \mathbb{Z}}\left(\lim _{n, F} H^{-i}\left(X, W_{n} \Omega_{X}^{j+2 m}\right)\right) \otimes_{W, \iota} \mathbb{C} \Rightarrow \operatorname{TP}_{i+j}(X) \otimes_{W, \iota} \mathbb{C}
$$

converging to the periodic topological cyclic homology of $X$. We call this spectral sequence the Hodge spectral sequence. The differentials in the spectral sequence preserve the direct sum decomposition of the $E^{2}$-term, and we call the number $w=j+m$ the weight 3 B comparing the two spectral sequences and using that determinants are multiplicative on exact sequences, we conclude from Berthelot's cohomological interpretation that

$$
\zeta(X, s)=\frac{\operatorname{det}\left(\mathrm{id}-q^{-s} \operatorname{Fr}_{q}^{*} \mid \mathrm{TP}_{1}(X) \otimes_{W, \iota} \mathbb{C}\right)}{\operatorname{det}\left(\mathrm{id}-q^{-s} \operatorname{Fr}_{q}^{*} \mid \operatorname{TP}_{0}(X) \otimes_{W, \iota} \mathbb{C}\right)}
$$

We wish to instead express the zeta function by using the full infinite dimensional graded $\mathbb{C}$-algebra $\operatorname{TP}_{*}(X) \otimes_{W, \iota} \mathbb{C}$. Indeed, we contend that this theory precisely is the infinite dimensional theory produced by Deninger 15 through an algebraic modification of the finite dimensional crystalline cohomology theory employing the Riemann-Hilbert correspondance on $\mathbb{G}_{m}$. Accordingly, as a consequence of op. cit., Corollary 2.8, we conclude that with $\Theta$ as in Theorem $\AA$

$$
\zeta(X, s)=\frac{\operatorname{det}_{\infty}\left(s \cdot \mathrm{id}-\Theta \mid \mathrm{TP}_{\mathrm{od}}(X) \otimes_{W, \iota} \mathbb{C}\right)}{\operatorname{det}_{\infty}\left(s \cdot \mathrm{id}-\Theta \mid \mathrm{TP}_{\mathrm{ev}}(X) \otimes_{W, \iota} \mathbb{C}\right)}
$$

This completes the outline of the proof of Theorem $\mathrm{A}$. We remark that this result gives a cohomological interpretation of the zeta function $\zeta(X, s)$ itself as opposed to one of the function $Z(X, t)$ with $\zeta(X, s)=Z\left(X, q^{-s}\right)$.

We close with a remark concerning periodicity. If $k$ is a commutative ring, then the Hochschild homology $\mathrm{HH}_{*}(k)$ is an anticommutative graded $k$-algebra and the graded ideal $I \subset \mathrm{HH}_{*}(k)$ spanned by the homogeneous elements of positive even

\footnotetext{
${ }^{2}$ Bhatt-Morrow-Scholze [3 have now constructed a filtration of TP by weight, the filtration quotients of which are given by crystalline cohomology.
} 
degree has a natural divided power structure [12, Exposé 7]. In particular, it is not possible for the canonical $k$-algebra homomorphism from the symmetric algebra

$$
S_{k}\left(\mathrm{HH}_{2}(k)\right) \longrightarrow \mathrm{HH}_{*}(k)
$$

to be an isomorphism, unless $k$ is a $\mathbb{Q}$-algebra. The topological Hochschild homology $\mathrm{THH}_{*}(k)$ also is an anticommutative graded $k$-algebra, but there is no longer a divided power structure on graded ideal $I \subset \mathrm{THH}_{*}(k)$ spanned by homogeneous elements of positive even degree. Hence, the canonical $k$-algebra homomorphism

$$
S_{k}\left(\mathrm{THH}_{2}(k)\right) \longrightarrow \mathrm{THH}_{*}(k)
$$

can be an isomorphism, and Bökstedt's periodicity theorem 8 shows that this is indeed the case for $k=\mathbb{F}_{p}$; see also [26. Theorem 5.2]. It is this basic periodicity theorem in conjunction with the cyclotomic structure of topological Hochschild homology that makes Theorem $\mathrm{A}$ possible.

\section{The Tate spectrum}

In this section, we recall Greenlees' generalization of Tate cohomology $\mathbf{2 0}$. We refer the reader to [26, Section 4] for more details and will follow the conventions therein. A more modern and comprehensive account, which in particular treats the multiplicative properties of the construction in detail, is given in $\mathbf{3 2}$.

Let $G$ be a compact Lie group, let $E$ be a free left $G$-CW-complex whose underlying space is contractible, and let $f: E_{+} \rightarrow S^{0}$ be the map that collapses $E$ onto $0 \in\{0, \infty\}=S^{0}$. We consider the following cofibration sequence

$$
E_{+} \stackrel{f}{\longrightarrow} S^{0} \stackrel{i}{\longrightarrow} \tilde{E} \stackrel{\partial}{\longrightarrow} \Sigma E_{+}
$$

in the homotopy category of pointed left $G$-spaces. The sequence is unique, up to unique isomorphism, and the induced sequence of suspension $G$-spectra is a cofibration sequence in the $G$-stable homotopy category. We abuse notation and denote the latter sequence by the same symbols. Now, for $X$ a $G$-spectrum, we consider the diagram

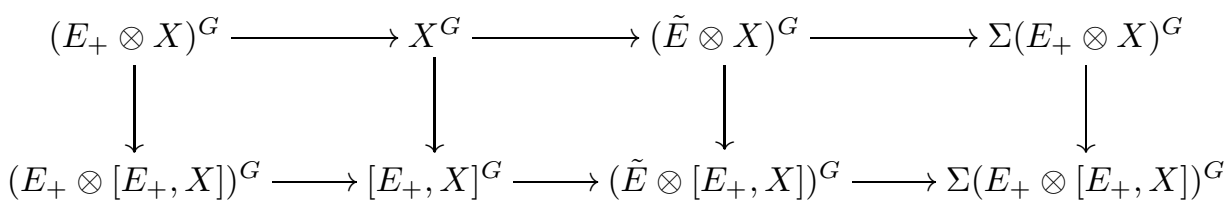

where we write " $\otimes$ " and "[-, $]$ " to indicate the symmetric monoidal product and internal hom object in the homotopy category of $G$-spectra, respectively. The lefthand vertical morphism is an isomorphism. We write the lower sequence as

$$
H .\left(G, S^{\mathfrak{g}} \otimes X\right) \stackrel{N}{\longrightarrow} H^{\cdot}(G, X) \stackrel{i}{\longrightarrow} \hat{H}^{\cdot}(G, X) \stackrel{\partial}{\longrightarrow} \Sigma H .\left(G, S^{\mathfrak{g}} \otimes X\right)
$$

and call it the Tate cofibration sequence. Here $S^{\mathfrak{g}}$ is the one-point compactification of the adjoint representation of $G$ on its Lie algebra $\mathfrak{g}$.

We are interested mainly in the case, where $G$ is either the full circle group $\mathbb{T}$ of complex numbers of modulus 1 or one of its finite subgroups $C_{r} \subset \mathbb{T}$. We record the following result, referring the reader to [11] for background on $p$-completion. 
Lemma 1.1. Let $p$ be a prime number and let $X$ be a $\mathbb{T}$-spectrum. If the underlying spectrum of $X$ is p-complete and bounded below, then the canonical morphism

$$
\hat{H}^{\cdot}(\mathbb{T}, X) \longrightarrow \operatorname{holim}_{n} \hat{H}^{\cdot}\left(C_{p^{n}}, X\right)
$$

is a weak equivalence.

Proof. It is proved in [24, Lemma 2.1.1] that the morphism in question becomes a weak equivalence after $p$-completion. Hence, it suffices to show that the domain and target of this morphism are $p$-complete. We prove the statement for the domain; the proof for the target is analogous. We wish to show that for every integer $i$, the canonical morphism

$$
\pi_{i}(\hat{H} \cdot(\mathbb{T}, X)) \longrightarrow \pi_{i}\left(\hat{H}^{\cdot}(\mathbb{T}, X), \mathbb{Z}_{p}\right)
$$

is an isomorphism. The assumption that the underlying spectrum of $X$ is bounded below implies that there exists a non-negative integer $s$ depending on $i$ such that the morphism

$$
\left(\operatorname{Fil}_{s}(\tilde{E}) \otimes\left[E_{+}, X\right]\right)^{\mathbb{T}} \longrightarrow\left(\tilde{E} \otimes\left[E_{+}, X\right]\right)^{\mathbb{T}}
$$

induced by the inclusion of the $s$-skeleton induces an isomorphism of homotopy groups in degrees less than or equal to $i$. We may further assume that the $\mathbb{T}$-CWcomplex $\operatorname{Fil}_{s}(\tilde{E})$ is finite. Therefore, replacing $X$ by $\operatorname{Fil}_{s}(\tilde{E}) \otimes X$, the underlying spectrum of which again is $p$-complete, it suffices to show that the group cohomology spectrum $H^{\cdot}(\mathbb{T}, X)$ is $p$-complete. In this case, the completion morphism factors as the composition

$$
H^{\cdot}(\mathbb{T}, X) \longrightarrow H^{\cdot}\left(\mathbb{T}, X_{p}\right) \longrightarrow H^{\cdot}(\mathbb{T}, X)_{p}
$$

of the morphism induced by the completion morphism for $X$ and a canonical isomorphism. But the left-hand morphism is a weak equivalence by the assumption that the underlying non-equivariant spectrum of $X$ is $p$-complete.

The skeleton filtrations of the pointed $G$-CW-complexes $E_{+}$and $\tilde{E}$ give rise to spectral sequences converging conditionally to the homotopy groups of the three terms in the Tate cofibration sequence. We call the spectral sequence converging to the homotopy groups of the Tate spectrum the Tate spectral sequence. In the situation of Lemma 1.1, we take $E=S\left(\mathbb{C}^{\infty}\right)$ and $\tilde{E}=S^{\mathbb{C}^{\infty}}$ with the $G$-CWstructures defined in [27, Section 4.4]. For the circle group $G=\mathbb{T}$, the spectral sequence takes the form

$$
E^{2}=S\left\{t^{ \pm 1}\right\} \otimes \pi_{*}(X) \Rightarrow \hat{H}^{-*}(\mathbb{T}, X)
$$

with the generator $t=c_{1}(\mathcal{O}(1)) \in E_{-2,0}^{2}$ specified below. For $G=C_{p^{n}}$, we have

$$
E^{2}=S\left\{t^{ \pm 1}\right\} \otimes \pi_{*}(X) / p^{n} \pi_{*}(X) \Rightarrow \hat{H}^{-*}\left(C_{p^{n}}, X\right),
$$

if the homotopy groups $\pi_{*}(X)$ are $p$-torsion free, and

$$
E^{2}=\Lambda\{u\} \otimes S\left\{t^{ \pm 1}\right\} \otimes \pi_{*}(X) \Rightarrow \hat{H}^{-*}\left(C_{p^{n}}, X\right),
$$

if the homotopy groups $\pi_{*}(X)$ are annihilated by $p$. We define the generators $t \in E_{-2,0}^{2}$ and $u \in E_{-1,0}^{2}$ to be the classes of the cycles specified in [27, Lemma 4.2.1]. We now define the generator $t$ for $G=\mathbb{T}$ to be the unique class that restricts to the generator $t$ for $G=C_{p^{n}}$. If $X$ is a ring $\mathbb{T}$-spectrum, then the spectral sequences are 
multiplicative with the indicated bi-graded ring structure on the $E^{2}$-term, except that if $p=2$ and $n=1$, then $u^{2}=t$. The restriction maps in Tate cohomology

$$
\hat{H}^{\cdot}(\mathbb{T}, X) \longrightarrow \hat{H}^{\cdot}\left(C_{p^{n}}, X\right) \longrightarrow \hat{H}^{\cdot}\left(C_{p^{n-1}}, X\right)
$$

induce multiplicative maps of Tate spectral sequences that, on the $E^{2}$-terms, are given by the tensor product of the canonical projections

$$
\pi_{*}(X) \longrightarrow \pi_{*}(X) / p^{n} \pi_{*}(X) \longrightarrow \pi_{*}(X) / p^{n-1} \pi_{*}(X)
$$

and the graded ring homomorphisms that take $t$ to $t$ and $u$ to 0 .

\section{Cyclotomic spectra}

We briefly recall the notion of a $p$-cyclotomic spectrum, which was introduced in 26. Section 2] and codifies the structure available on the topological Hochschild spectrum in addition to the circle action discovered by Connes. A better and more flexible version of this notion was introduced by Nikolaus and Scholze [32.

The group $\mathbb{T}$ has the special property that there exists a group isomorphism

$$
\mathbb{T} \stackrel{\rho}{\longrightarrow} \mathbb{T} / C_{p},
$$

which we will always choose to be the $p$ th root. A $p$-cyclotomic structure on a $\mathbb{T}$-spectrum $T$ was defined in [26, Definition 2.2] to be a morphism of $\mathbb{T}$-spectra

$$
\rho^{*}(\tilde{E} \otimes T)^{C_{p}} \stackrel{r}{\longrightarrow} T
$$

with the property that for all $n \geqslant 0$, the induced map of $C_{p^{n}}$-fixed point spectra is a weak equivalence. The diagram at the beginning of Section 1 takes the form

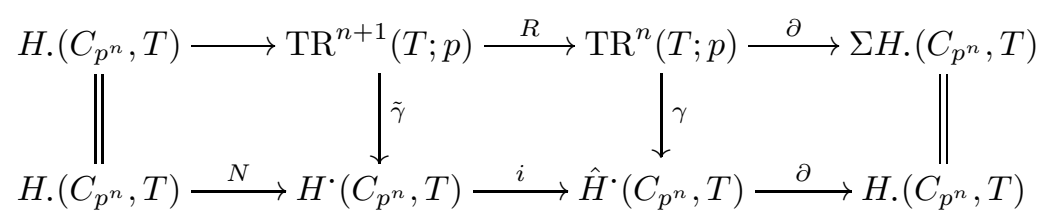

where $\operatorname{TR}^{n}(T ; p)$ is the $C_{p^{n-1}}$-fixed point spectrum of $T$, and where the morphism

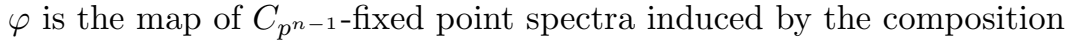

$$
T \stackrel{r}{\longleftarrow} \rho^{*}(\tilde{E} \otimes T)^{C_{p}} \stackrel{f^{*}}{\longrightarrow} \rho^{*}\left(\tilde{E} \otimes\left[E_{+}, T\right]\right)^{C_{p}}
$$

of an inverse of the morphism $r$ and the morphism $f^{*}$ in the original diagram. We remark that, recently, Nikolaus and Scholze have made the remarkable observation that it is only the composite $\rho$-equivariant morphism

$$
T \stackrel{\gamma}{\longrightarrow} \hat{H}^{\cdot}\left(C_{p}, T\right)
$$

that is of importance and that the factorization of this map inherent in the original definition of a $p$-cyclotomic structure is immaterial. As demonstrated in $\mathbf{3 2}$, this approach makes it possible to develop the theory of cyclotomic spectra without the use of equivariant stable homotopy theory. In the following, we will therefore refer to $\varphi$ as the cyclotomic structure morphism. We call the morphism

$$
\mathrm{TR}^{n+1}(T ; p) \stackrel{R}{\longrightarrow} \mathrm{TR}^{n}(T ; p)
$$


in the diagram above the restriction morphism; and we call the morphism

$$
\operatorname{TR}^{n+1}(T ; p) \stackrel{F}{\longrightarrow} \operatorname{TR}^{n}(T ; p)
$$

given by the canonical inclusion the Frobenius morphism. We write

$$
\mathrm{TF}(T ; p)=\operatorname{holim}_{n, F} \operatorname{TR}^{n}(T ; p)
$$

for the homotopy limit with respect to the Frobenius morphisms. Using the $p$ cyclotomic structure on $T$, we now define the inverse Frobenius operator

$$
\mathrm{TF}(T ; p) \stackrel{\varphi^{-1}}{\longrightarrow} \mathrm{TF}(T ; p)
$$

to be the composition

$$
\operatorname{holim}_{n, F} \mathrm{TR}^{n}(T ; p) \longrightarrow \operatorname{holim}_{n, F} \mathrm{TR}^{n+1}(T ; p) \longrightarrow \operatorname{holim}_{n, F} \mathrm{TR}^{n}(T ; p)
$$

of the restriction along the successor functor and the morphism induced by the restriction morphisms. The cyclotomic structure morphism induces a morphism

$$
\mathrm{TF}(T ; p)=\operatorname{holim}_{n, F} \operatorname{TR}^{n}(T ; p) \stackrel{\gamma}{\longrightarrow} \operatorname{holim}_{n, F} \hat{H}^{\cdot}\left(C_{p^{n}}, T\right),
$$

and Lemma 1.1 identifies the target with $\hat{H}^{\bullet}(\mathbb{T}, T)$, provided that $T$ is $p$-complete and bounded below. The inverse Frobenius operator $\varphi^{-1}$ does typically not extend to an operator defined on $\hat{H}^{\cdot}(\mathbb{T}, T)$, even at the level of homotopy groups.

\section{Topological Hochschild homology}

In this section, we recall the structure of topological Hochschild homology for schemes smooth over a perfect field of characteristic $p>0$ from [21. We also recall Bökstedt's periodicity theorem from $\mathbf{8}$.

Topological Hochschild homology is defined in the same generality as algebraic $K$-theory and assigns to an exact category with weak equivalences a cyclotomic spectrum. In particular, for a scheme $X$, applying this theory to the exact category of perfect complexes of quasi-coherent $\mathcal{O}_{X}$-modules with quasi-isomorphisms as weak equivalences, defines a cyclotomic spectrum $\mathrm{THH}(X)$. The derived tensor product of perfect complexes gives rise to a pairing on $\mathrm{THH}(X)$, which makes it a commutative cyclotomic ring spectrum 3 Moreover, for every morphism of schemes $f: X \rightarrow Y$, left derived extension of scalars gives rise to a morphism of cyclotomic ring spectra $f^{*}: \operatorname{THH}(Y) \rightarrow \operatorname{THH}(X)$; and for every proper morphism of schemes $f: X \rightarrow Y$, right derived restriction of scalars gives rise to a morphism of cyclotomic $\operatorname{THH}(Y)$-module spectra $f_{*}: \operatorname{THH}(X) \rightarrow \operatorname{THH}(Y)$.

The homotopy groups $\mathrm{THH}_{*}(X)$ form an anticommutative graded ring and we define $\mathrm{THH}_{*}\left(\mathcal{O}_{X}\right)$ to be the sheaf of anticommutative graded rings on the small étale site of $X$ associated to the presheaf that to an étale morphism $f: U \rightarrow X$ assigns $\mathrm{THH}_{*}(U)$. There is a natural ring isomorphism

$$
\mathcal{O}_{X} \stackrel{\eta}{\longrightarrow} \operatorname{THH}_{0}\left(\mathcal{O}_{X}\right)
$$

\footnotetext{
${ }^{3}$ It follows from 32 Section IV.2] that $\mathrm{THH}(X)$ is an $\mathbb{E}_{\infty}$-algebra in cyclotomic spectra.
} 
given by the inclusion of the zero-skeleton, and we will consider $\operatorname{THH}_{*}\left(\mathcal{O}_{X}\right)$ as a sheaf of anticommutative graded $\mathcal{O}_{X}$-algebras via this map. As such, it is quasicoherent by [17, Proposition 3.2.1], and there is a multiplicative and conditionally convergent descent spectral sequence

$$
E_{i, j}^{2}=H^{-i}\left(X, \operatorname{THH}_{j}\left(\mathcal{O}_{X}\right)\right) \Rightarrow \mathrm{THH}_{i+j}(X) .
$$

We consider this spectral sequence, which we call the Hodge spectral sequence for topological Hochschild homology, in detail in Section 6 below. Since $\mathrm{THH}_{j}\left(\mathcal{O}_{X}\right)$ is zero for $j<0$, it shows, in particular, that $\operatorname{THH}_{i}(X)$ is zero for $i<-\operatorname{dim}(X)$.

More generally, we let $r=p^{n-1}$ be a prime power and let $C_{r} \subset \mathbb{T}$ be the subgroup of order $r$. The equivariant homotopy groups

$$
\operatorname{TR}_{*}^{n}(X ; p)=\pi_{*}\left(\operatorname{THH}(X)^{C_{r}}\right)
$$

again form an anticommutative graded ring and we define $\operatorname{TR}_{*}^{n}\left(\mathcal{O}_{X} ; p\right)$ to be the sheaf of anticommutative graded rings on the small étale site of $X$ associated to the presheaf that to an étale morphism $f: U \rightarrow X$ assigns $\operatorname{TR}_{*}^{n}(U ; p)$. By [26, Theorem $\mathrm{F}]$, there is a canonical natural ring isomorphism

$$
W_{n}\left(\mathcal{O}_{X}\right) \stackrel{\eta}{\longrightarrow} \operatorname{TR}_{0}^{n}\left(\mathcal{O}_{X} ; p\right),
$$

which is compatible with the restriction, Frobenius, and Verschiebung operators, and we view $\operatorname{TR}_{*}^{n}\left(\mathcal{O}_{X} ; p\right)$ as a sheaf of anticommutative graded $W_{n}\left(\mathcal{O}_{X}\right)$-algebras via this map. As such, it is quasi-coherent. Indeed, this follows from the proof of [28, Proposition 6.2.4] by using [9, Theorem B] and [10, Corollary 15.4], which were proved later.

The $W_{n}\left(\mathcal{O}_{X}\right)$-modules $\operatorname{TR}_{j}^{n}\left(\mathcal{O}_{X} ; p\right)$ together with the cup product $\cup$, the restriction $R$, the Frobenius $F$, the Verschiebung $V$, and Connes' operator $d$ constitute a $p$-typical Witt complex over $\mathcal{O}_{X}$; see [25, Definition 4.1]. Accordingly, this $p$-typical Witt complex receives a unique map of $p$-typical Witt complexes

$$
W_{n} \Omega_{X}^{j} \stackrel{\eta}{\longrightarrow} \operatorname{TR}_{j}^{n}\left(\mathcal{O}_{X} ; p\right)
$$

from the $p$-typical de Rham-Witt complex of $X$, which, by definition, is the initial $p$-typical Witt complex over $\mathcal{O}_{X}$. This map is an isomorphism for $j \leqslant 1$ by $[\mathbf{2 2}$.

We next recall the following fundamental periodicity theorem. The basic case $n=1$ is Bökstedt's theorem proved in his paper $[\mathbf{8}$ which unfortunately remains unpublished. A detailed outline of Bökstedt's proof is given in [26, Section 5.2], and the proof of the general case $n \geqslant 1$ is given in [26. Theorem 5.5].

TheOREM 3.1. Let $k$ be a perfect field of characteristic $p>0$ and let $n$ be $a$ positive integer. The canonical map of graded $W_{n}(k)$-algebras

$$
S_{W_{n}(k)}\left(\mathrm{TR}_{2}^{n}(k ; p)\right) \longrightarrow \mathrm{TR}_{*}^{n}(k ; p)
$$

is an isomorphism and the $W_{n}(k)$-module $\mathrm{TR}_{2}^{n}(k ; p)$ is free of rank 1 .

In Section 4, we define a preferred $W_{n}(k)$-module generator $\alpha_{n} \in \mathrm{TR}_{2}^{n}(k ; p)$, the divided Bott element. It satisfies $F\left(\alpha_{n}\right)=\alpha_{n-1}$ and $R\left(\alpha_{n}\right)=p \cdot \alpha_{n-1}$, showing that, in [26. Theorem 5.5], we can arrange for the units $\lambda_{n}$ to be equal to 1 .

We also recall from [21, Theorem B] the following result, which we will use in Section 6 below to construct the Hodge spectral sequence mentioned in the Introduction. 
TheOREM 3.2. Let $k$ be a perfect field of characteristic $p>0$, let $n$ be a positive integer, and let $f: X \rightarrow \operatorname{Spec}(k)$ be a smooth morphism. In this situation, the canonical map of graded $W_{n}\left(\mathcal{O}_{X}\right)$-algebras

$$
W_{n} \Omega_{X}^{*} \otimes_{f^{*} W_{n}(k)} f^{*} \mathrm{TR}_{*}^{n}(k ; p) \longrightarrow \mathrm{TR}_{*}^{n}\left(\mathcal{O}_{X} ; p\right)
$$

is an isomorphism.

We note that, by comparison, if $k$ is a separably closed field of any characteristic and if $f: X \rightarrow \operatorname{Spec}(k)$ is a smooth morphism, then the canonical map

$$
K_{*}^{M}\left(\mathcal{O}_{X}\right) \otimes_{f^{*} \mathbb{Z} / p^{n} \mathbb{Z}} f^{*} K_{*}\left(k, \mathbb{Z} / p^{n} \mathbb{Z}\right) \longrightarrow K_{*}\left(\mathcal{O}_{X}, \mathbb{Z} / p^{n} \mathbb{Z}\right)
$$

is an isomorphism by Voevodsky. If $\operatorname{char}(k) \neq p$, then $K_{*}\left(k, \mathbb{Z} / p^{n} \mathbb{Z}\right)$ is a symmetric algebra generated by the Bott element $\beta_{\epsilon, n} \in K_{2}\left(k, \mathbb{Z} / p^{n} \mathbb{Z}\right)$ by Suslin [33, 34]; and if $\operatorname{char}(k)=p$, then $K_{*}\left(k, \mathbb{Z} / p^{n} \mathbb{Z}\right)=\mathbb{Z} / p^{n} \mathbb{Z}$ by Geisser-Levine $\mathbf{1 8}$.

\section{The divided Bott element}

Let $k$ be a perfect field of characteristic $p>0$. We proved in [26, Section 5] that, as an anticommutative graded ring $\mathrm{TP}_{*}(k)=S_{W(k)}\left\{v^{ \pm 1}\right\}$ for some $v \in \mathrm{TP}_{-2}(k)$. In this section, we make a preferred choice of the generator $v \in \mathrm{TP}_{-2}(k)$ based on the results in 24. We call this generator the inverse divided Bott element.

We first define the ring isomorphism

$$
W(k) \stackrel{\tau}{\longrightarrow} \mathrm{TP}_{0}(k)
$$

by which we consider $\operatorname{TP}_{*}(k)$ a graded $W(k)$-algebra. Since $k$ is an $\mathbb{F}_{p^{-}}$algebra, the Witt vector Frobenius $F: W_{n}(k) \rightarrow W_{n-1}(k)$ is equal to the common composite of the maps in the following diagram with the horizontal maps induced by the Frobenius $\varphi: k \rightarrow k$,

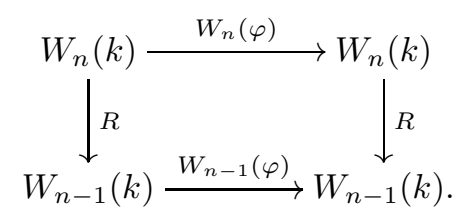

Therefore, we may define a ring homomorphism

$$
\lim _{n, F} W_{n}(k) \stackrel{\varphi^{\infty}}{\longrightarrow} \lim _{n, R} W_{n}(k)=W(k)
$$

to be the map of limits induced by the map of limit systems that, at level $n$, is given by the ring homomorphism $W_{n}\left(\varphi^{n}\right): W_{n}(k) \rightarrow W_{n}(k)$. The map $\varphi^{\infty}$ is an isomorphism, because $k$ is perfect. Moreover, the composition

$$
W_{n}(k) \stackrel{\eta}{\longrightarrow} \mathrm{TR}_{0}^{n}(k ; p) \stackrel{\gamma}{\longrightarrow} \hat{H}^{0}\left(C_{p^{n}}, \mathrm{THH}(k)\right)
$$

of the canonical isomorphism from [26, Theorem $\mathrm{F}$ ] and the isomorphism induced by the cyclotomic structure map defines a ring isomorphism

$$
\lim _{n, F} W_{n}(k) \stackrel{\hat{\eta}}{\longrightarrow} \lim _{n, F} \hat{H}^{0}\left(C_{p^{n}}, \operatorname{THH}(k)\right),
$$

where the structure maps in the right-hand limit system are the restriction maps in Tate cohomology. Finally, we have an isomorphism of graded rings

$$
\mathrm{TP}_{*}(k) \stackrel{\rho}{\longrightarrow} \lim _{n, F} \hat{H}^{-*}\left(C_{p^{n}}, \operatorname{THH}(k)\right),
$$


which is also given by restriction maps in Tate cohomology. We now define the ring isomorphism $\tau$ to be the composite $\rho^{-1} \circ \hat{\eta} \circ\left(\varphi^{\infty}\right)^{-1}$. Extension of scalars along the unique ring homomorphism $f: \mathbb{F}_{p} \rightarrow k$ defines a map of graded $W(k)$-algebras

$$
W(k) \otimes_{W\left(\mathbb{F}_{p}\right)} \mathrm{TP}_{*}\left(\mathbb{F}_{p}\right) \longrightarrow \mathrm{TP}_{*}(k)
$$

and that this map is an isomorphism.

We proceed to define the generator $v \in \mathrm{TP}_{-2}\left(\mathbb{F}_{p}\right)$, and, to this end, we recall the main results of [24]. Let $C$ be the perfectoid field of $p$-adic complex numbers. It is defined, we recall, to be the completion of an algebraic closure of the field $\mathbb{Q}_{p}$ of $p$-adic numbers and is both complete and algebraically closed. Its valuation ring $\mathcal{O}_{C}$ is a complete valuation ring with value group $\mathbb{Q}$, the residue field $k$ of which is an algebraic closure of $\mathbb{F}_{p}$.

We first recall the results on the algebraic $K$-theory of $\mathcal{O}_{C}$ proved by Suslin in [33, 34, based on the rigidity theorems of Gabber [16] and Gillet-Thomason [19. The group $K_{j}\left(\mathcal{O}_{C}\right)$ is $p$-divisible if $j$ is positive, and it is uniquely $p$-divisible if $j$ is both positive and even. Hence, the Bockstein homomorphism

$$
K_{2}\left(\mathcal{O}_{C}, \mathbb{Z}_{p}\right) \stackrel{\partial}{\longrightarrow} T_{p}\left(K_{1}\left(\mathcal{O}_{C}\right)\right)=\operatorname{Hom}_{\mathbb{Z}}\left(\mathbb{Q}_{p} / \mathbb{Z}_{p}, K_{1}\left(\mathcal{O}_{C}\right)\right)
$$

from the $p$-adic $K$-group in degree 2 to the $p$-primary Tate module of the $K$-group in degree 1 is an isomorphism. The resulting map of graded $K_{0}\left(\mathcal{O}_{C}, \mathbb{Z}_{p}\right)$-algebras

$$
S_{K_{0}\left(\mathcal{O}_{C}, \mathbb{Z}_{p}\right)}\left(T_{p}\left(K_{1}\left(\mathcal{O}_{C}\right)\right)\right) \longrightarrow K_{*}\left(\mathcal{O}_{C}, \mathbb{Z}_{p}\right),
$$

moreover, is an isomorphism. Here the ring $K_{0}\left(\mathcal{O}_{C}, \mathbb{Z}_{p}\right)$ is canonically isomorphic to $\mathbb{Z}_{p}$ and the $p$-primary Tate module $\mathbb{Z}_{p}(1)=T_{p}\left(K_{1}\left(\mathcal{O}_{C}\right)\right)$ is a free $\mathbb{Z}_{p}$-module of rank 1. An element $\epsilon \in \mathbb{Z}_{p}(1)$ determines and is determined by the sequence $\left(\epsilon^{(v)}\right)_{v \geqslant 0}$ of compatible $p$-power roots of unity in $C$ defined by $\epsilon^{(v)}=\epsilon\left(p^{-v}+\mathbb{Z}_{p}\right)$ and it is a generator if and only if the $p$ th root of unity $\epsilon^{(1)}$ is primitive. In this situation, we follow Thomason [36] and define the Bott element associated with $\epsilon$ to be the unique generator

$$
\beta_{\epsilon} \in K_{2}\left(\mathcal{O}_{C}, \mathbb{Z}_{p}\right)
$$

such that $\partial\left(\beta_{\epsilon}\right)=\epsilon$.

We next recall the corresponding results for TF-theory from [24, which, as it turns out, are completely analogous. The group $\mathrm{TF}_{j}\left(\mathcal{O}_{C} ; p\right)$ is $p$-divisible if $j$ is positive, and it is uniquely $p$-divisible if $j$ is both positive and even. Accordingly, the Bockstein homomorphism induces an isomorphism

$$
\mathrm{TF}_{2}\left(\mathcal{O}_{C} ; p, \mathbb{Z}_{p}\right) \stackrel{\partial}{\longrightarrow} T_{p}\left(\mathrm{TF}_{1}\left(\mathcal{O}_{C} ; p\right)\right)=\operatorname{Hom}_{\mathbb{Z}}\left(\mathbb{Q}_{p} / \mathbb{Z}_{p}, \mathrm{TF}_{1}\left(\mathcal{O}_{C} ; p\right)\right),
$$

and it is proved in op. cit. that the resulting map of graded $\operatorname{TF}_{0}\left(\mathcal{O}_{C} ; p, \mathbb{Z}_{p}\right)$-algebras

$$
S_{\mathrm{TF}_{0}\left(\mathcal{O}_{C} ; p, \mathbb{Z}_{p}\right)}\left(T_{p}\left(\mathrm{TF}_{1}\left(\mathcal{O}_{C} ; p\right)\right)\right) \longrightarrow \mathrm{TF}_{*}\left(\mathcal{O}_{C} ; p, \mathbb{Z}_{p}\right)
$$

is an isomorphism. Moreover, the ring $\operatorname{TF}_{0}\left(\mathcal{O}_{C} ; p, \mathbb{Z}_{p}\right)$ is canonically isomorphic to Fontaine's ring of $p$-adic periods

$$
A_{\text {inf }}=\lim _{n, F} W_{n}\left(\mathcal{O}_{C}\right),
$$

which plays a prominent role in $p$-adic Hodge theory. The $p$-primary Tate module

$$
A_{\text {inf }}\{1\}=T_{p}\left(\operatorname{TF}_{1}\left(\mathcal{O}_{C} ; p\right)\right)=\lim _{n, F} T_{p}\left(W_{n} \Omega_{\mathcal{O}_{C}}^{1}\right)
$$


is a free $A_{\mathrm{inf}}$-module of rank 1 . However, the image by the cyclotomic trace map

$$
K_{*}\left(\mathcal{O}_{C}, \mathbb{Z}_{p}\right) \stackrel{\operatorname{tr}}{\longrightarrow} \mathrm{TF}_{*}\left(\mathcal{O}_{C} ; p, \mathbb{Z}_{p}\right)
$$

of the Bott element $\beta_{\epsilon}$ is not a generator of this $A_{\text {inf }}$-module. Instead, we proved in [24, Theorem B] that the image of the Bott element, which, by abuse of notation, we also denote by $\beta_{\epsilon}$, is divisible by the non-unit

$$
\mu_{\epsilon}=[\varepsilon]-1=\left(\left[\epsilon^{(n)}\right]_{n}-1\right)_{n \geqslant 1} \in \lim _{n, F} W_{n}\left(\mathcal{O}_{C}\right)=A_{\text {inf }}
$$

and that the element $\alpha_{\epsilon}=\mu_{\epsilon}^{-1} \cdot \beta_{\epsilon}$ is a generator of $\operatorname{TF}_{2}\left(\mathcal{O}_{C} ; p, \mathbb{Z}_{p}\right)$.

Definition 4.1. If $\epsilon$ is a generator of the free $\mathbb{Z}_{p}$-module $\mathbb{Z}_{p}(1)$, then the associated divided Bott element is the generator

$$
\alpha_{\epsilon}=\mu_{\epsilon}^{-1} \cdot \beta_{\epsilon}
$$

of the free $A_{\text {inf }}$-module $\mathrm{TF}_{2}\left(\mathcal{O}_{C} ; p, \mathbb{Z}_{p}\right)=A_{\text {inf }}\{1\}$.

By functoriality, the left action of the Galois group $G=\operatorname{Gal}\left(C / \mathbb{Q}_{p}\right)$ on $\mathcal{O}_{C}$ induces an action on the graded ring $\mathrm{TF}_{*}\left(\mathcal{O}_{C} ; p, \mathbb{Z}_{p}\right)$, and the action on the Bott element is given by

$$
\sigma \cdot \beta_{\epsilon}=\beta_{\sigma(\epsilon)}=\chi(\sigma) \cdot \beta_{\epsilon},
$$

where $\chi: G \rightarrow \operatorname{Aut}\left(\mu_{p \infty}\right)=\mathbb{Z}_{p}^{*}$ is the cyclotomic character. Since $A_{\text {inf }}$ is an integral domain, we therefore conclude that

$$
\sigma \cdot \alpha_{\epsilon}=\alpha_{\sigma(\epsilon)}=\chi(\sigma) \cdot \sigma\left(\mu_{\epsilon}\right)^{-1} \cdot \mu_{\epsilon} \cdot \alpha_{\epsilon} .
$$

Similarly, the inverse Frobenius operator induces a homorphism of graded rings

$$
\mathrm{TF}_{*}\left(\mathcal{O}_{C} ; p, \mathbb{Z}_{p}\right) \stackrel{\varphi^{-1}}{\longrightarrow} \mathrm{TF}_{*}\left(\mathcal{O}_{C} ; p, \mathbb{Z}_{p}\right) .
$$

In degree zero, it is given, up to canonical isomorphism, by the ring automorphism

$$
A_{\text {inf }} \stackrel{\varphi^{-1}}{\longrightarrow} A_{\text {inf }}
$$

defined as the composition

$$
\lim _{n, F} W_{n}\left(\mathcal{O}_{C}\right) \stackrel{\operatorname{res}_{S}}{\longrightarrow} \lim _{n, F} W_{n+1}\left(\mathcal{O}_{C}\right) \stackrel{\lim _{n, F} R}{\longrightarrow} \lim _{n, F} W_{n}\left(\mathcal{O}_{C}\right)
$$

of the restriction along the successor functor and the map induced by the Witt vector restriction. The cyclotomic trace map takes values in the sub-graded ring of elements fixed by the inverse Frobenius operator. In particular,

$$
\varphi^{-1}\left(\beta_{\epsilon}\right)=\beta_{\epsilon},
$$

and using again that $A_{\text {inf }}$ is an integral domain, we therefore conclude that

$$
\varphi^{-1}\left(\alpha_{\epsilon}\right)=\varphi^{-1}\left(\xi_{\epsilon}\right) \cdot \alpha_{\epsilon},
$$

where $\xi_{\epsilon} \in A_{\text {inf }}$ is the element defined by

$$
\xi_{\epsilon}=\varphi\left(\mu_{\epsilon}\right) \cdot \mu_{\epsilon}^{-1}=1+[\epsilon]+[\epsilon]^{2}+\cdots+[\epsilon]^{p-1} .
$$

We remark that this element is a generator of the kernel of the ring homomorphism

$$
A_{\text {inf }} \stackrel{\theta}{\longrightarrow} \mathcal{O}_{C}
$$

which, in our formulation, is given by the canonical projection from the limit that defines the left-hand ring to the term $n=1$. 
We recall from [27, Addendum 5.4.4] and [24, Proposition 2.1.4] that the map of graded $A_{\text {inf-algebras induced by the cyclotomic structure map }}$

$$
\mathrm{TF}_{*}\left(\mathcal{O}_{C} ; p, \mathbb{Z}_{p}\right) \stackrel{\gamma}{\longrightarrow} \mathrm{TP}_{*}\left(\mathcal{O}_{C}, \mathbb{Z}_{p}\right)
$$

is an isomorphism in non-negative degrees. Hence, it becomes an isomorphism after inverting the divided Bott element $\alpha_{\epsilon}$. We now define

$$
v_{\epsilon} \in \mathrm{TP}_{-2}\left(\mathcal{O}_{C}, \mathbb{Z}_{p}\right)
$$

to be the inverse of the image of the divided Bott element $\alpha_{\epsilon}$. It is represented in the Tate spectral sequence by the element $t \in E_{-2,0}^{2}$ defined in Section 1. Clearly, the Galois group $G$ acts on the inverse divided Bott element by

$$
\sigma \cdot v_{\epsilon}=v_{\sigma(\epsilon)}=\chi(\sigma)^{-1} \cdot \sigma\left(\mu_{\epsilon}\right) \cdot \mu_{\epsilon}^{-1} \cdot v_{\epsilon} .
$$

Moreover, the inverse Frobenius operator $\varphi^{-1}$ on $\mathrm{TF}_{*}\left(\mathcal{O}_{C} ; p, \mathbb{Z}_{p}\right)$ gives rise to a meromorphic Frobenius operator

$$
\mathrm{TP}_{*}\left(\mathcal{O}_{C}, \mathbb{Z}_{p}\right) \stackrel{\varphi}{\longrightarrow} \mathrm{TP}_{*}\left(\mathcal{O}_{C}, \mathbb{Z}_{p}\right),
$$

which is defined and invertible away from the divisor " $\xi_{\epsilon}=0$ " and satisfies

$$
\varphi\left(v_{\epsilon}\right)=\xi_{\epsilon} \cdot v_{\epsilon} .
$$

This completes our recollection of the results from $2 \mathbf{2 4}$.

We consider the ring homomorphism

$$
A_{\text {inf }} \stackrel{i}{\longrightarrow} W(k)
$$

defined to be the composition

$$
\lim _{n, F} W_{n}\left(\mathcal{O}_{C}\right) \longrightarrow \lim _{n, F} W_{n}(k) \stackrel{\varphi^{\infty}}{\longrightarrow} \lim _{n, R} W_{n}(k)
$$

of the map induced by the canonical projection of $\mathcal{O}_{C}$ onto its residue field $k$ and the isomorphism $\varphi^{\infty}$ above. Extension of scalars along $i$ induces an isomorphism

$$
W(k) \otimes_{A_{\text {inf }}} \mathrm{TP}_{*}\left(\mathcal{O}_{C}, \mathbb{Z}_{p}\right) \stackrel{i^{*}}{\longrightarrow} \mathrm{TP}_{*}\left(k, \mathbb{Z}_{p}\right),
$$

the target of which is canonically isomorphic to $\mathrm{TP}_{*}(k)$.

Proposition 4.2. The class $v=i^{*}\left(v_{\epsilon}\right) \in \mathrm{TP}_{-2}(k)$ is independent of the choice of generator $\epsilon \in \mathbb{Z}_{p}(1)$ and descends to a generator $v \in \mathrm{TP}_{-2}\left(\mathbb{F}_{p}\right)$. Moreover, the meromorphic Frobenius operator

$$
\mathrm{TP}_{*}(k) \stackrel{\varphi}{\longrightarrow} \mathrm{TP}_{*}(k)
$$

is defined and invertible away from the divisor " $p=0$ " and satisfies $\varphi(v)=p \cdot v$.

Proof. If $\epsilon \in \mathbb{Z}_{p}(1)$ is a fixed generator, then a general generator is of the form $\sigma(\epsilon) \in \mathbb{Z}_{p}(1)$, for some $\sigma \in G$. So it suffices to show that $i: A_{\text {inf }} \rightarrow W(k)$ maps the unit $u_{\epsilon}=\chi(\sigma)^{-1} \cdot \sigma\left(\mu_{\epsilon}\right) \cdot \mu_{\epsilon}^{-1} \in A_{\text {inf }}$ to $1 \in W(k)$. We may assume that $\chi(\sigma)$ is an integer, in which case, the image $u_{\epsilon, n}$ of $u_{\epsilon}$ by the canonical projection from $A_{\text {inf }}$ to $W_{n}\left(\mathcal{O}_{C}\right)$ is given by

$$
\begin{aligned}
u_{\epsilon, n} & =\chi(\sigma)^{-1} \cdot\left(\left[\sigma\left(\epsilon^{(n)}\right)\right]_{n}-1\right) \cdot\left(\left[\epsilon^{(n)}\right]_{n}-1\right)^{-1} \\
& =\chi(\sigma)^{-1} \cdot\left(\left[\epsilon^{(n)}\right]_{n}^{\chi(\sigma)}-1\right) \cdot\left(\left[\epsilon^{(n)}\right]_{n}-1\right)^{-1} \\
& =\chi(\sigma)^{-1} \cdot \sum_{0 \leqslant j<\chi(\sigma)}\left[\epsilon^{(n)}\right]_{n}^{j} .
\end{aligned}
$$


But the canonical projection from $\mathcal{O}_{C}$ to $k$ takes $\epsilon^{(n)}$ to 1 , and hence, the induced map from $W_{n}\left(\mathcal{O}_{C}\right)$ to $W_{n}(k)$ takes $u_{\epsilon, n}$ to 1 , as desired. This shows that the class

$$
v=i^{*}\left(v_{\epsilon}\right) \in \mathrm{TP}_{-2}(k)
$$

is independent of $\epsilon$ as stated. It also shows that this class is Galois fixed, and hence, that it descends to a class $v \in \mathrm{TP}_{-2}\left(\mathbb{F}_{p}\right)$. Similarly, we have

$$
i\left(\xi_{\epsilon}\right)=p,
$$

which proves the remaining statements.

Remark 4.3. It follows from Proposition 4.2 that if we define $\alpha_{n} \in \mathrm{TR}_{2}^{n}\left(\mathbb{F}_{p} ; p\right)$ to be the image of $v^{-1} \in \mathrm{TF}_{2}\left(\mathbb{F}_{p} ; p\right)$, then $F\left(\alpha_{n}\right)=\alpha_{n-1}$ and $R\left(\alpha_{n}\right)=p \cdot \alpha_{n-1}$. This shows that, in [26. Proposition 5.4], we can arrange for the units $\lambda_{n}$ to be equal to 1.

\section{The conjugate spectral sequence}

We recall from the paper [30] the higher Cartier isomorphism and the structure of the conjugate spectral sequence converging to the crystalline cohomology of a scheme smooth and proper over a perfect field of positive characteristic.

Let $k$ be a perfect field of characteristic $p>0$ and let $f: X \rightarrow \operatorname{Spec}(k)$ be a smooth morphism. The comparison theorem [29, Théorème II.1.4] gives a canonical isomorphism of $W_{n}(k)$-modules

$$
H_{\text {crys }}^{i}\left(X / W_{n}(k)\right) \longrightarrow H^{i}\left(X, W_{n} \Omega_{X}^{\cdot}\right)
$$

from the crystalline cohomology of $X / W_{n}(k)$ defined in $[2$ to the hypercohomology of $X$ with coefficients in the de Rham-Witt complex. The second spectral sequence of hypercohomology gives a spectral sequence of $W_{n}(k)$-modules

$$
E_{2}^{i, j}=H^{i}\left(X, \mathcal{H}^{j}\left(W_{n} \Omega_{X}\right)\right) \Rightarrow H_{\text {crys }}^{i+j}\left(X / W_{n}(k)\right) .
$$

Being located in the first quadrant, it converges strongly. If $f: X \rightarrow \operatorname{Spec}(k)$ is also proper, and hence of finite relative dimension $d$, then the $W_{n}(k)$-modules $E_{2}^{i, j}$ are finitely generated by [29. Proposition II.2.1]. Therefore, taking limits with respect to the restriction maps, we obtain a spectral sequence of $W(k)$-modules

$$
E_{2}^{i, j}=\lim _{n, \mathcal{H}^{j}(R)} H^{i}\left(X, \mathcal{H}^{j}\left(W_{n} \Omega_{X}\right)\right) \Rightarrow H_{\text {crys }}^{i+j}(X / W(k)) .
$$

Indeed, by finite generation, the limit systems satisfy the Mittag-Leffler condition. To rewrite the $E_{2}$-term we recall the higher Cartier isomorphism.

We have isomorphisms of sheaves of abelian groups

$$
W_{n} \Omega_{X}^{j} \stackrel{R^{n}}{\longleftarrow} W_{2 n} \Omega_{X}^{j} /\left(V^{n} W_{n} \Omega_{X}^{j}+d V^{n} W_{n} \Omega_{X}^{j-1}\right) \stackrel{F^{n}}{\longrightarrow} \mathcal{H}^{j}\left(W_{n} \Omega_{X}^{\cdot}\right) .
$$

Indeed, the left-hand map is an isomorphism for formal reasons, and the right-hand map is an isomorphism by [30, Proposition III.1.4]. The inverse higher Cartier operator is defined to be the composite isomorphism

$$
C^{-n}=F^{n} \circ R^{-n} .
$$


It follows easily from the definition that the diagram of sheaves of abelian groups

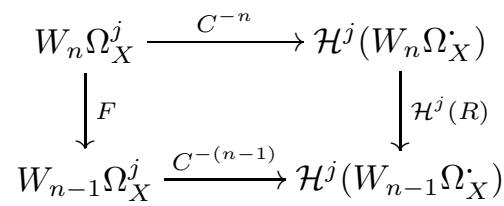

commutes, and hence, we obtain an induced isomorphism of abelian groups

$$
\lim _{n, F} H^{-i}\left(X, W_{n} \Omega_{X}^{j}\right) \longrightarrow \lim _{n, \mathcal{H}^{j}(R)} H^{-i}\left(X, \mathcal{H}^{j}\left(W_{n} \Omega_{X}\right)\right) .
$$

In addition, this map is an isomorphism of $W(k)$-modules, provided that we consider its domain to be a $W(k)$-module via the inverse of the isomorphism

$$
\lim _{n, F} W_{n}(k) \stackrel{\varphi^{\infty}}{\longrightarrow} \lim _{n, R} W_{n}(k)=W(k)
$$

defined in Section 4. We record these recollections from [29, 30] in the following result.

THEOREM 5.1. If $k$ is a perfect field of characteristic $p>0$ and $f: X \rightarrow \operatorname{Spec}(k)$ a smooth and proper morphism, then there is a spectral sequence of $W(k)$-modules

$$
E_{2}^{i, j}=\lim _{n, F} H^{i}\left(X, W_{n} \Omega_{X}^{j}\right) \Rightarrow H_{\mathrm{crys}}^{i+j}(X / W(k))
$$

which converges strongly.

We call the spectral sequence in Theorem 5.1 the conjugate spectral sequence. The analysis of its $E_{2}$-term in [30, Théorème III.2.2] gives the following result.

Corollary 5.2. Let $k$ be a finite field of order $q=p^{r}$, let $W$ be its ring of $p$-typical Witt vectors, let $\iota: W \rightarrow \mathbb{C}$ be an embedding, and let $f: X \rightarrow \operatorname{Spec}(k)$ be a smooth and proper morphism. In this situation, there is a strongly convergent spectral sequence of degree-wise finite dimensional $\mathbb{C}$-vector spaces

$$
E_{2}^{i, j}=\left(\lim _{n, F} H^{i}\left(X, W_{n} \Omega_{X}^{j}\right)\right) \otimes_{W, \iota} \mathbb{C} \Rightarrow H_{\text {crys }}^{i+j}(X / W) \otimes_{W, \iota} \mathbb{C} .
$$

Proof. The spectral sequence in the statement is obtained from the conjugate spectral sequence by extension of scalars along the ring homomorphism $\iota: W \rightarrow \mathbb{C}$, which is flat. Finally, by [30. Théorème III.2.2], each of the $W(k)$-modules $E_{2}^{i, j}$ in the conjugate spectral sequence is finitely generated modulo torsion.

REMARK 5.3. In the situation of Theorem [5.1, [30, Théorème III.2.2] also shows that the torsion sub- $W(k)$-module of $\lim _{n, F} H^{i}\left(X, W_{n} \Omega_{X}^{j}\right)$ is annihilated by $p$. However, this torsion sub-W(k)-module may well not be finitely generated 4 as the example of a supersingular K3 surface shows; see [30, IV.2.15.6] and [29, II.7.2].

\section{The Hodge spectral sequence}

In this section, we construct the Hodge spectral sequence for periodic topological cyclic homology of schemes smooth and proper over a perfect field of characteristic $p>0$.

In general, let $X$ be a site, let $F$ be a presheaf of spectra on $X$, and let

$$
F(U) \stackrel{\eta_{U}}{\longrightarrow} H^{\cdot}(U, F)
$$

\footnotetext{
${ }^{4}$ The weight spectral sequence of [3] does not have this pathology.
} 
be the canonical morphism to the associated cohomology presheaf. The latter may be defined by means of the Godement construction, if the site $X$ has enough points, and by a fibrant replacement in an appropriate model structure, in general; see [36, 31. In either case, there is a hypercohomology spectral sequence

$$
E_{i, j}^{2}=H^{-i}\left(U, \pi_{j}(F)^{\sim}\right) \Rightarrow H^{-i-j}(U, F)
$$

from the cohomology of $U$ with coefficients in the sheaves assoaciated with the presheaves of homotopy groups which converges conditionally to the homotopy groups of the global sections of the cohomology presheaf. If the morphism $\eta_{U}$ is a weak equivalence for every object $U$ of $X$, then we say that the presheaf $F$ satisfies descent. In this case, the abutment of the hypercohomlogy spectral sequence is canonically identified with $\pi_{i+j}(F(U))$.

If $X$ is a scheme, then by [17, Definition 3.2.3] or [5, Theorem 1.3] the presheaf of spectra on the small étale site of $X$ that to an étale morphism $h: U \rightarrow X$ assigns $\operatorname{THH}(U)$ satisfies descent. Hence, in this situation the hypercohomology spectral sequence takes the form

$$
E_{i, j}^{2}=H^{-i}\left(U, \mathrm{THH}_{j}\left(\mathcal{O}_{X}\right)\right) \Rightarrow \mathrm{THH}_{i+j}(U),
$$

where, as in Section $3 . \mathrm{THH}_{j}\left(\mathcal{O}_{X}\right)$ is the sheaf of $\mathcal{O}_{X}$-modules on the small étale site of $X$ associated with the presheaf that to an étale morphism $h: U \rightarrow X$ assigns $\mathrm{THH}_{j}(U)$. We recall from [17, Proposition 3.2.1] that this $\mathcal{O}_{X}$-module is quasicoherent. In particular, in the case where $U$ is affine, the spectral sequence collapses and the edge homomorphism

$$
\mathrm{THH}_{j}(U) \longrightarrow H^{0}\left(U, \mathrm{THH}_{j}\left(\mathcal{O}_{X}\right)\right)
$$

is an isomorphism.

We next consider the presheaf of spectra on the small étale site of $X$ that to an étale morphism $h: U \rightarrow X$ assigns the Tate spectrum

$$
F(U)=\hat{H}^{\cdot}\left(C_{p^{n}}, \operatorname{THH}(U)\right),
$$

and let $\hat{H}^{-j}\left(C_{p^{n}}, \operatorname{THH}\left(\mathcal{O}_{X}\right)\right)$ be the sheaf of $W_{n}\left(\mathcal{O}_{X}\right)$-modules on the small étale site of $X$ that to an étale morphism $h: U \rightarrow X$ assigns $\hat{H}^{-j}\left(C_{p^{n}}, \mathrm{THH}(U)\right)$.

Lemma 6.1. Let $k$ be a perfect field of characteristic $p>0$. If $f: k \rightarrow A$ is a smooth morphism of relative dimension $d$, then the Tate spectral sequence

$$
E^{2}=\hat{H}^{-i}\left(C_{p^{n}}, \operatorname{THH}_{j}(A)\right) \Rightarrow \hat{H}^{-i-j}\left(C_{p^{n}}, \operatorname{THH}(A)\right)
$$

converges strongly and $E_{i, j}^{\infty}$ vanishes for $j \geqslant d+2 n$. In particular, the induced filtration of the abutment is of finite length.

Proof. The spectral sequence in question takes the form

$$
E^{2}=\Lambda\left\{u_{n}\right\} \otimes S\left\{t^{ \pm 1}, \alpha\right\} \otimes \Omega_{A}^{*} \Rightarrow \hat{H}^{-*}\left(C_{p^{n}}, \operatorname{THH}(A)\right),
$$

where the classes $u_{n}, t$, and $\alpha$ have bidegrees $(-1,0),(-2,0)$, and $(0,2)$, and where $\Omega_{A}^{*}$ is located in bidegrees $(0, j)$ with $0 \leqslant j \leqslant d$. By [26, Lemma 5.4], the classes $t$ and $\alpha$ are infinite cycles, and if $A=k$, then

$$
d^{2 n+1}\left(u_{n}\right)=\lambda \cdot t^{n+1} \alpha^{n}
$$

for some unit $\lambda \in \mathbb{F}_{p}$. By comparison, the $\mathbb{T}$-Tate spectral sequence takes the form

$$
E^{2}=S\left\{t^{ \pm 1}, \alpha\right\} \otimes \Omega_{A}^{*} \Rightarrow \hat{H}^{-*}(\mathbb{T}, \operatorname{THH}(A)),
$$


and the inclusion of $C_{p^{n}}$ in $\mathbb{T}$ induces a map from the latter spectral sequence to the form, which, on $E^{2}$-terms, is induced by the unit map $\eta: k \rightarrow \Lambda\left\{u_{n}\right\}$. Now, for degree reasons, all nonzero differentials in the latter spectral sequence are even. Therefore, in the former spectral sequence, the $d^{2 n+1}$-differential is given by

$$
d^{2 n+1}\left(u_{n} \otimes \omega\right)=\lambda \cdot t^{n+1} \alpha^{n} \omega .
$$

This shows that $E_{i, j}^{2 n+2}$ vanishes for $j \geqslant d+2 n$, and hence, so does $E_{i, j}^{\infty}$. Finally, since the Tate spectral sequence always converges conditionally, we conclude that, in the situation at hand, it converges strongly; see [6. Theorem 7.1].

Proposition 6.2. Let $k$ be a perfect field of characteristic $p>0$, let $n$ be $a$ positive integer, and let $f: k \rightarrow A$ be a smooth morphism. If $h: A \rightarrow B$ is an étale morphism, then the induced map of graded $W_{n}(B)$-algebras

$$
W_{n}(B) \otimes_{W_{n}(A)} \hat{H}^{-*}\left(C_{p^{n}}, \operatorname{THH}(A)\right) \longrightarrow \hat{H}^{-*}\left(C_{p^{n}}, \operatorname{THH}(B)\right)
$$

is an isomorphism.

Proof. The Tate spectral sequence is a spectral sequence of $W_{n+1}(A)$-modules

$$
E_{i, j}^{2}=\hat{H}^{-i}\left(C_{p^{n}}, F_{*}^{n} \mathrm{THH}_{j}(A)\right) \Rightarrow R_{*} \hat{H}^{-i-j}\left(C_{p^{n}}, \mathrm{THH}(A)\right),
$$

where $F_{*}^{n}(-)$ and $R_{*}(-)$ indicates the restriction of scalars along the Frobenius $F^{n}: W_{n+1}(A) \rightarrow A$ and restriction $R: W_{n+1}(A) \rightarrow W_{n}(A)$, respectively. We recall from [9. Theorem B] that the morphism $W_{n+1}(h): W_{n+1}(A) \rightarrow W_{n+1}(B)$ again is étale and hence flat. Therefore, by extension of scalars along this morphism, we obtain a spectral sequence of $W_{n+1}(B)$-modules

$$
\begin{aligned}
E_{i, j}^{2} & =\hat{H}^{-i}\left(C_{p^{n}}, W_{n+1}(B) \otimes_{W_{n+1}(A)} F_{*}^{n} \operatorname{THH}_{j}(A)\right) \\
& \Rightarrow W_{n+1}(B) \otimes_{W_{n+1}(A)} R_{*} \hat{H}^{-i-j}\left(C_{p^{n}}, \mathrm{THH}(A)\right),
\end{aligned}
$$

and Lemma 6.1 shows that both this spectral sequence and the spectral sequence

$$
E_{i, j}^{2}=\hat{H}^{-i}\left(C_{p^{n}}, F_{*}^{n} \mathrm{THH}_{j}(B)\right) \Rightarrow R_{*} \hat{H}^{-i-j}\left(C_{p^{n}}, \mathrm{THH}(B)\right),
$$

converge strongly. The morphism $h: A \rightarrow B$ induces a map of spectral sequences from the former to the latter, and we claim that this map is an isomorphism. To prove this, it suffices to show that the induced map of $W_{n+1}(B)$-modules

$$
W_{n+1}(B) \otimes_{W_{n+1}(A)} F_{*}^{n} \mathrm{THH}_{j}(A) \longrightarrow F_{*}^{n} \mathrm{THH}_{j}(B)
$$

is an isomorphism. If $n=0$, then this is proved in [17, Proposition 3.2.1], and the general case follows from this case and from the fact proved in [10. Corollary 15.4] that the left-hand diagram below is a cocartesian square of commutative rings.
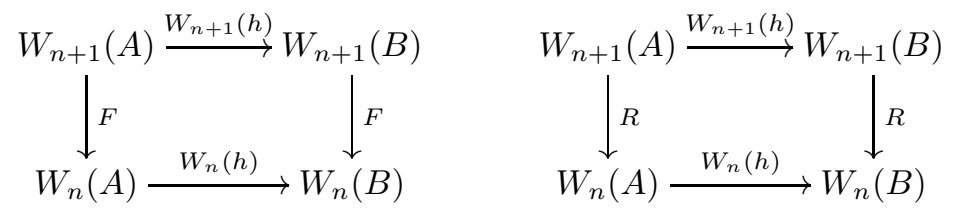

We therefore conclude that the map of graded $W_{n+1}(B)$-modules

$$
W_{n+1}(B) \otimes_{W_{n+1}(A)} R_{*} \hat{H}^{-*}\left(C_{p^{n}}, \operatorname{THH}(A)\right) \longrightarrow R_{*} \hat{H}^{-*}\left(C_{p^{n}}, \mathrm{THH}(B)\right)
$$


induced by $h: A \rightarrow B$ is an isomorphism. Finally, by loc. cit., the right-hand diagram above also is a cocartesian square of commutative rings. This shows that the map in the statement is an isomorphism as desired.

COROLlary 6.3. Let $k$ be a perfect field of characteristic $p>0$, let $n$ be a positive integer, and let $f: X \rightarrow \operatorname{Spec}(k)$ be a smooth morphism of finite relative dimension. In this situation, the graded $W_{n}\left(\mathcal{O}_{X}\right)$-algebra $\hat{H}^{-*}\left(C_{p^{n}}, \operatorname{THH}\left(\mathcal{O}_{X}\right)\right)$ is quasi-coherent.

Proof. As proved in [1, Exposé VII, Proposition 3.1], the étale morphisms $h: A \rightarrow B$ with $\operatorname{Spec}(A)$ open in $X$ generate the small étale topos of $X$. Therefore, the statement follows from Proposition 6.2 .

Proposition 6.4. Let $k$ be a perfect field of characteristic $p>0$, let $n$ be a positive integer, and let $f: X \rightarrow \operatorname{Spec}(k)$ is a smooth morphism of finite relative dimension. In this situation, the presheaf of spectra on the small étale site of $X$ that to an étale morphism $h: U \rightarrow X$ assigns $\hat{H}^{\cdot}\left(C_{p^{n}}\right.$, $\left.\mathrm{THH}(U)\right)$ satisfies descent.

Proof. Let again $F$ denote the presheaf in question. We wish to prove that for every étale morphism $h: U \rightarrow X$, the canonical morphism

$$
F(U) \stackrel{\eta_{U}}{\longrightarrow} H^{\cdot}(U, F)
$$

is a weak equivalence. Corollary 6.3 shows that, for $U$ affine, the sheafification map

$$
\pi_{j}(F(U)) \longrightarrow H^{0}\left(U, \pi_{j}(F)^{\sim}\right)
$$

is an isomorphism and that the descent spectral sequence

$$
E_{i, j}^{2}=H^{-i}\left(X, \pi_{j}(F)^{\sim}\right) \Rightarrow H^{-i-j}(U, F)
$$

collapses. But the sheafification map is equal to the composition of the map of homotopy groups induced by $\eta_{U}$ and the edge homomorphism of the descent spectral sequence, so we conclude that $\eta_{U}$ is a weak equivalence in this case. If $h: U \rightarrow X$ is a general étale morphism, then we choose a hyper-covering $\mathcal{U}$ of $U$ by affine schemes étale over $U$ and consider the diagram in which the two vertical morphisms are the canonical morphisms to the respective Čech cohomology spectra,

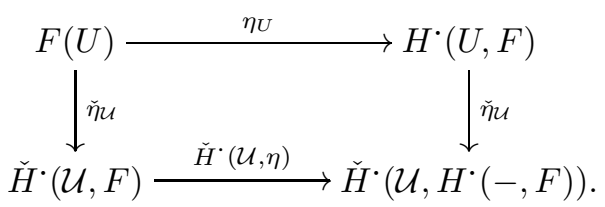

The lower horizontal morphism is a weak equivalence by the case already considered, and the right-hand vertical morphism is a weak equivalence, since sheaf cohomology satisfies descent. We claim that also the left-hand vertical morphism is a weak equivalence. We write this morphism as the composition

$$
\hat{H}^{\cdot}\left(C_{p^{n}}, \operatorname{THH}(U)\right) \longrightarrow \hat{H}^{\cdot}\left(C_{p^{n}}, \check{H}^{\cdot}(\mathcal{U}, \mathrm{THH}(-))\right) \longrightarrow \check{H}^{\cdot} \cdot\left(\mathcal{U}, \hat{H}^{\cdot}\left(C_{p^{n}}, \mathrm{THH}(-)\right)\right)
$$

of the morphism in Tate cohomlogy induced by the corresponding morphism in topological Hochschild homology and the canonical map from Tate cohomology of a homotopy limit to the homotopy limit of the Tate cohomology of the individual terms in the limit system. The former map is a weak equivalence, since topological Hochschild homology satisfies descent, and the latter map is a weak equivalence, 
since the spectra in the limit system are universally bounded below by the relative dimension of $f: X \rightarrow \operatorname{Spec}(k)$.

Remark 6.5. We do not know whether or not Corollary6.3 and Proposition6.4 hold for all schemes $X$.

We view the next result as an analogue of analytic continuation.

Proposition 6.6. Let $k$ be a perfect field of characteristic $p>0$, let $n \geqslant 1$ be an integer, and let $f: X \rightarrow \operatorname{Spec}(k)$ is a smooth morphism of relative dimension $d$. In this situation, the map of graded $W_{n}\left(\mathcal{O}_{X}\right)$-algebras

$$
\operatorname{TR}_{i}^{n}\left(\mathcal{O}_{X} ; p\right) \stackrel{\gamma}{\longrightarrow} \hat{H}^{-i}\left(C_{p^{n}}, \operatorname{THH}\left(\mathcal{O}_{X}\right)\right)
$$

induced by the cyclotomic structure morphism is an isomorphism for $i \geqslant d$.

Proof. The domain and target $W_{n}\left(\mathcal{O}_{X}\right)$-modules of the map in question both are quasi-coherent. Therefore, it suffices to take $X=\mathbb{A}_{k}^{d}$ and show that the induced map of global sections is an isomorphism for $i \geqslant d$. Moreover, by [37, Theorem 2.4], it suffices to consider the case $n=1$. Now, in the case $d=0$, the result was proved in [26. Proposition 5.3]; see also loc. cit., Remark 5.5. In general, we have an equivalence of $C_{p}$-spectra

$$
\operatorname{THH}(k) \otimes N^{\mathrm{cy}}\left(\left\langle x_{1}, \ldots, x_{d}\right\rangle\right) \stackrel{\alpha}{\longrightarrow} \mathrm{THH}\left(k\left[x_{1}, \ldots, x_{d}\right]\right)
$$

where the second factor on the left-hand side is the cyclic bar-construction of the free commutative monoid on the indicated generators. We refer to [23. Proposition 3] for the definition of the map $\alpha$ and for the proof that it is a weak equivalence. Moreover, there is a commutative diagram of spectra

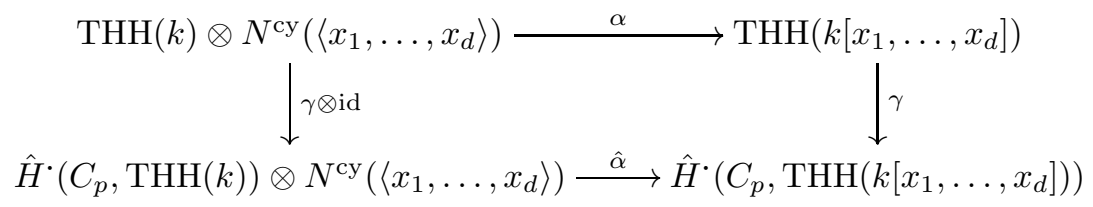

and the lower horizontal map also is a weak equivalence; see [26, Corollary 9.1]. We claim that the reduced singular homology of $N^{\mathrm{cy}}\left(\left\langle x_{1}, \ldots, x_{d}\right\rangle\right)$ is concentrated in the degrees $0 \leqslant i \leqslant d$. Granting this, we conclude from the case $d=0$ that the left-hand vertical map in the diagram above induces an isomorphism of homotopy groups in degrees greater than or equal to $d$. But then the same holds for the right-hand vertical map, which proves the proposition. To prove the claim we use that there are canonical isomorphisms

$$
\Omega_{\mathbb{Z}\left[x_{1}, \ldots, x_{d}\right] / \mathbb{Z}}^{i} \longrightarrow \mathrm{HH}_{i}\left(\mathbb{Z}\left[x_{1}, \ldots, x_{d}\right] / \mathbb{Z}\right) \longrightarrow \tilde{H}_{i}\left(N^{\mathrm{cy}}\left(\left\langle x_{1}, \ldots, x_{d}\right\rangle\right), \mathbb{Z}\right)
$$

and the easy calculation of the left-hand side.

TheOREM 6.7. Let $k$ be a perfect field of characteristic $p>0$, let $n$ be a positive integer, and let $f: X \rightarrow \operatorname{Spec}(k)$ be a smooth morphism of finite relative dimension. In this situation, the canonical map of graded $W_{n}\left(\mathcal{O}_{X}\right)$-algebras

$$
W_{n} \Omega_{X}^{*} \otimes_{f^{*} W_{n}(k)} f^{*} \hat{H}^{-*}\left(C_{p^{n}}, \operatorname{THH}(k)\right) \stackrel{\hat{\eta}}{\longrightarrow} \hat{H}^{-*}\left(C_{p^{n}}, \operatorname{THH}\left(\mathcal{O}_{X}\right)\right)
$$

is an isomorphism. 
Proof. We consider the commutative diagram of graded $W_{n}\left(\mathcal{O}_{X}\right)$-algebras in which the horizontal maps are the canonical maps and the vertical maps are induced by the cyclotomic structure maps,

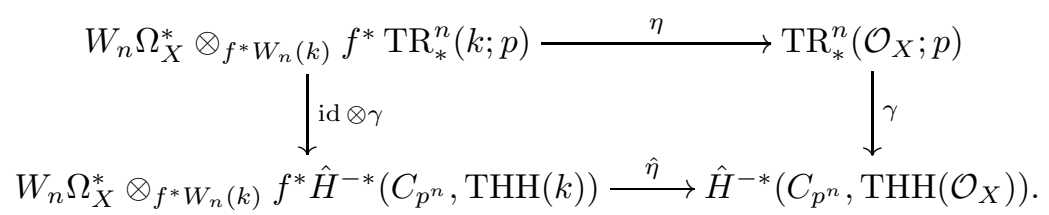

We recall from [21, Theorem B] that the top horizontal map is an isomorphism and from Proposition 6.6 that the right-hand vertical map is an isomorphism in degrees greater than or equal to the relative dimension of $f: X \rightarrow \operatorname{Spec}(k)$. Since the graded $W_{n}\left(\mathcal{O}_{X}\right)$-algebras in the bottom row both are 2-periodic, the theorem follows.

THEOREM 6.8. If $X$ is a scheme smooth and proper morphism over a perfect field $k$ of characteristic $p>0$, then there is a spectral sequence of $W(k)$-modules

$$
E_{i, j}^{2}=\bigoplus_{m \in \mathbb{Z}} \lim _{n, F} H^{-i}\left(X, W_{n} \Omega_{X}^{j+2 m}\right) \Rightarrow \mathrm{TP}_{i+j}(X)
$$

which converges strongly.

Proof. We first argue that the canonical morphism

$$
\operatorname{TP}(X)=\hat{H}^{\cdot}(\mathbb{T}, \operatorname{THH}(X)) \longrightarrow \operatorname{holim}_{n, F} \hat{H}^{\cdot}\left(C_{p^{n}}, \operatorname{THH}(X)\right)
$$

is a weak equivalence. It follows from [24, Lemma 2.1.1] that this morphism becomes a weak equivalence after $p$-completion, and therefore, it suffices to show that the domain and target are $p$-complete. This, in turn, follows from the definition of the Tate spectrum, once we shows that $\operatorname{THH}(X)$ is $p$-complete and bounded below. That the spectrum $\operatorname{THH}(X)$ is $p$-complete is an immediate consequence of its homotopy groups being $k$-vector spaces, and hence, annihilated by $p$. To see that it is bounded below, we consider the conditionally convergent descent spectral sequence of $k$-vector spaces

$$
E_{i, j}^{2}=H^{-i}\left(X, \mathrm{THH}_{j}\left(\mathcal{O}_{X}\right)\right) \Rightarrow \mathrm{THH}_{i+j}(X) .
$$

Since the structure morphism $f: X \rightarrow \operatorname{Spec}(k)$ is smooth and proper, its relative dimension $d$ is finite. Therefore, the $k$-vector space $E_{i, j}^{2}$ is zero, unless $-d \leqslant i \leqslant 0$ and $j \geqslant 0$, which shows that the spectral sequence converges strongly and that $\mathrm{THH}_{j}(X)$ is zero for $j<-d$. So the canonical morphism at the beginning of the proof is a weak equivalence as desired.

Next, by Proposition 6.4 we have descent spectral sequences

$$
E_{i, j}^{2}=H^{-i}\left(X, \hat{H}^{-j}\left(C_{p^{n}}, \operatorname{THH}\left(\mathcal{O}_{X}\right)\right)\right) \Rightarrow \hat{H}^{-i-j}\left(C_{p^{n}}, \operatorname{THH}(X)\right),
$$

which converge strongly. Indeed, the spectral sequences converge conditionally, and $E_{i, j}^{2}$ is zero, unless $-d \leqslant i \leqslant 0$. Moreover, identifying

$$
\hat{H}^{-*}\left(C_{p^{n}}, \operatorname{THH}(k)\right)=S_{W_{n}(k)}\left\{v^{ \pm 1}\right\}
$$

with $v \in \mathrm{TP}_{-2}(k)$, Theorem 6.7 gives canonical isomorphisms of $W_{n}\left(\mathcal{O}_{X}\right)$-modules

$$
\bigoplus_{m \in \mathbb{Z}} W_{n} \Omega_{X}^{j+2 m} \longrightarrow \hat{H}^{-j}\left(C_{p^{n}}, \operatorname{THH}\left(\mathcal{O}_{X}\right)\right),
$$


and hence, we may rewrite the descent spectral sequences above in the form

$$
E_{i, j}^{2}=\bigoplus_{m \in \mathbb{Z}} H^{-i}\left(X, W_{n} \Omega_{X}^{j+2 m}\right) \Rightarrow \hat{H}^{-i-j}\left(C_{p^{n}}, \operatorname{THH}(X)\right) .
$$

We claim that the $W_{n}(k)$-module $E_{i, j}^{2}$ is finitely generated for all values of $(i, j)$. Indeed, since $f: X \rightarrow \operatorname{Spec}(k)$ is smooth and proper of relative dimension $d$, it follows from [29, Proposition II.2.1] that the $W_{n}(k)$-module $H^{-i}\left(X, W_{n} \Omega_{X}^{j+2 m}\right)$ is finitely generated, for all integers $m$. But unless $0 \leqslant j+2 m \leqslant d$, said $W_{n}(k)$-module is zero, so the claim follows. Moreover, for cohomological dimension reasons, $E_{i, j}^{2}$ is zero, unless $-d \leqslant i \leqslant 0$, so we conclude that also $\hat{H}^{-i}\left(C_{p^{n}}, \operatorname{THH}(X)\right)$ is a finitely generated $W_{n}(k)$-module, for all integers $i$. Hence, taking limits with respect to the Frobenius maps, we obtain a spectral sequence of $W(k)$-modules

$$
E_{i, j}^{2}=\bigoplus_{m \in \mathbb{Z}} \lim _{n, F} H^{-i}\left(X, W_{n} \Omega_{X}^{j+2 m}\right) \Rightarrow \lim _{n, F} \hat{H}^{-i-j}\left(C_{p^{n}}, \operatorname{THH}(X)\right),
$$

and the corresponding higher derived limits vanish. Indeed, by finite generation, the limit systems satisfy the Mittag-Leffler condition. This also implies that the canonical map

$$
\mathrm{TP}_{i}(X) \longrightarrow \lim _{n, F} \hat{H}^{-i}\left(C_{p^{n}}, \mathrm{THH}(X)\right)
$$

is an isomorphism, so the theorem follows.

COROllary 6.9. Let $k$ be a finite field of order $q=p^{r}$, let $W$ be its ring of $p$-typical Witt vectors, and let $\iota: W \rightarrow \mathbb{C}$ be a choice of embedding. If $X$ is a scheme smooth and proper over $k$, then there is a strongly convergent spectral sequence of degree-wise finite dimensional $\mathbb{C}$-vector spaces

$$
E_{i, j}^{2}=\bigoplus_{m \in \mathbb{Z}}\left(\lim _{n, F} H^{-i}\left(X, W_{n} \Omega_{X}^{j+2 m}\right)\right) \otimes_{W, \iota} \mathbb{C} \Rightarrow \mathrm{TP}_{i+j}(X) \otimes_{W, \iota} \mathbb{C} .
$$

Proof. We have the strongly convergent spectral sequence of $W$-modules

$$
E_{i, j}^{2}=\bigoplus_{m \in \mathbb{Z}} \lim _{n, F} H^{-i}\left(X, W_{n} \Omega_{X}^{j+2 m}\right) \Rightarrow \operatorname{TP}_{i+j}(X)
$$

from Theorem 6.8. For cohomological dimension reasons, it is concentrated in the strip $-d \leqslant i \leqslant 0$, where $d$ is the relative dimension of $X$ over $k$. Therefore, we obtain the desired spectral sequence by extension of scalars along the flat ring homomorphism $\iota: W \rightarrow \mathbb{C}$. Finally, by [30, Théorème III.2.2], we see that the $\mathbb{C}$-vector spaces $E_{i, j}^{2}$ are finite dimensional as in the proof of Corollary 5.2 .

\section{Regularized determinants}

In this section, we recall the definitions of regularized determinant and anomalous dimension following Deninger $\mathbf{1 5}$, and complete the proof of Theorem $\mathrm{A}$.

The definition requires a choice of principal branch $\operatorname{Arg}(\lambda)$ of the argument of $\lambda \in \mathbb{C}^{*}$. We follow [15, Section 1] and choose $-\pi<\operatorname{Arg}(\lambda) \leqslant \pi$ and define

$$
\lambda^{-s}=|\lambda|^{-s} e^{-i s \operatorname{Arg}(\lambda)}
$$

for $\lambda \in \mathbb{C}^{*}$ and $s \in \mathbb{C}$. Let $V$ be a $\mathbb{C}$-vector spact5 of at most countably infinite dimension and let $\Theta: V \rightarrow V$ be a $\mathbb{C}$-linear map such that:

(1) For every $\lambda \in \mathbb{C}$, the generalized eigenspace $V_{\lambda}$ of $\Theta: V \rightarrow V$ associated to the eigenvalue $\lambda$ is finite dimensional.

(2) The Dirichlet series $\sum_{\lambda \in \mathbb{C}^{*}} \operatorname{dim}_{\mathbb{C}}\left(V_{\lambda}\right) \lambda^{-s}$ converges absolutely for $\operatorname{Re}(s) \gg 0$ and admits a meromorphic continuation $\zeta_{\Theta}(s)$ to the halfplane $\operatorname{Re}(s)>-\epsilon$ for some $\epsilon>0$ which is holomorphic at $s=0$.

\footnotetext{
${ }^{5}$ There is no topology on $V$; in particular, it is not a Hilbert space.
} 
The anomalous dimension and regularized determinant of $\Theta: V \rightarrow V$ are now defined to be the complex numbers

$$
\begin{aligned}
& \operatorname{dim}_{\infty}(\Theta \mid V)=\operatorname{dim}_{\mathbb{C}}\left(V_{0}\right)+\zeta_{\bar{\Theta}}(0), \\
& \operatorname{det}_{\infty}(\Theta \mid V)= \begin{cases}e^{-\zeta_{\Theta}^{\prime}(0)} & \text { if } \operatorname{dim}_{\mathbb{C}}\left(V_{0}\right)=0, \\
0 & \text { if } \operatorname{dim}_{\mathbb{C}}\left(V_{0}\right)>0,\end{cases}
\end{aligned}
$$

where, in the top line, $\bar{\Theta}: V / V_{0} \rightarrow V / V_{0}$ is the map induced by $\Theta: V \rightarrow V$. If $V$ is finite dimensional, then $\operatorname{dim}_{\infty}(\Theta \mid V)=\operatorname{dim}_{\mathbb{C}}(V)$ and $\operatorname{det}_{\infty}(\Theta \mid V)=\operatorname{det}(\Theta \mid V)$. Moreover, if $\delta$ is a positive real number, then one has

$$
\operatorname{det}_{\infty}(\delta \cdot \Theta \mid V)=\delta^{\operatorname{dim}_{\infty}(\Theta \mid V)} \operatorname{det}_{\infty}(\Theta \mid V),
$$

as expected. More importantly, by [15. Lemma 1.2], given a commutative diagram

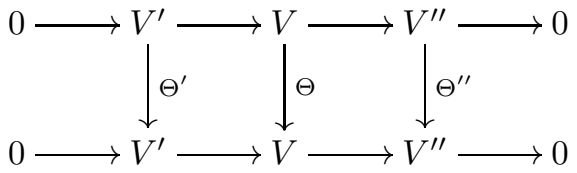

of $\mathbb{C}$-vector spaces and $\mathbb{C}$-linear maps with exact rows such that the right- and left-hand vertical maps satisfy (1)-(2), then so does the middle vertical map and

$$
\begin{aligned}
\operatorname{dim}_{\infty}(\Theta \mid V) & =\operatorname{dim}_{\infty}\left(\Theta^{\prime} \mid V^{\prime}\right)+\operatorname{dim}_{\infty}\left(\Theta^{\prime \prime} \mid V^{\prime \prime}\right) \\
\operatorname{det}_{\infty}(\Theta \mid V) & =\operatorname{det}_{\infty}\left(\Theta^{\prime} \mid V\right) \cdot \operatorname{det}_{\infty}\left(\Theta^{\prime \prime} \mid V^{\prime \prime}\right) .
\end{aligned}
$$

The following result is a special case of [15, Corollary 2.8].

Proposition 7.1. Let $T$ be an anticommutative graded $\mathbb{C}$-algebra such that the sub-C-Vector space $T_{j} \subset T$ of homogeneous elements of degree $j$ is finite dimensional for all integers $j$. Let $\Theta: T \rightarrow T$ be a graded $\mathbb{C}$-linear derivation and suppose that there exists a unit $v \in T_{-2}$ such that $\Theta(v)=\frac{2 \pi i}{\log q} \cdot v$. In this situation,

$$
\begin{aligned}
\operatorname{det}_{\infty}\left(s \cdot \mathrm{id}-\Theta \mid T_{2 *+j}\right) & =\operatorname{det}\left(\mathrm{id}-q^{-s \cdot \Theta} \mid T_{j}\right), \\
\operatorname{dim}_{\infty}\left(s \cdot \mathrm{id}-\Theta \mid T_{2 *+j}\right) & =0 .
\end{aligned}
$$

for all $s \in \mathbb{C}$ and for all integers $j$.

Remark 7.2. The two side of the equality in the statement of Proposition 7.1 only depend on the parity of the integer $j$. Indeed, we have the exact sequence

$$
0 \longrightarrow \frac{2 \pi i}{\log q} \mathbb{Z} \longrightarrow \mathbb{C} \longrightarrow \mathbb{C}^{*} \longrightarrow 0,
$$

where the right-hand map takes $\alpha \in \mathbb{C}$ to $q^{\alpha} \in \mathbb{C}^{*}$. In its most basic form, Deninger's formula expresses the inverse non-archimedean Euler factor $1-\lambda q^{-s}$ with $\lambda \in \mathbb{C}^{*}$ as the regularized product of the $s-\alpha$ with $\alpha \in \mathbb{C}$ ranging over all solutions to $q^{\alpha}=\lambda$.

Proof of Theorem A, By applying Proposition 7.1 to the anticommutative graded $\mathbb{C}$-algebra $\operatorname{TP}_{*}(X) \otimes_{W, \iota} \mathbb{C}$ and a graded $\mathbb{C}$-linear derivation

$$
\mathrm{TP}_{*}(X) \otimes_{W, \iota} \mathbb{C} \stackrel{\Theta}{\longrightarrow} \mathrm{TP}_{*}(X) \otimes_{W, \iota} \mathbb{C}
$$

as in the statement of the theorem, we find that for all $s \in \mathbb{C}$ and $j \in \mathbb{Z}$,

$$
\operatorname{det}_{\infty}\left(s \cdot \mathrm{id}-\Theta \mid \mathrm{TP}_{2 *+j}(X) \otimes_{W, \iota} \mathbb{C}\right)=\operatorname{det}\left(\mathrm{id}-q^{-s} \operatorname{Fr}_{q}^{*} \mid \mathrm{TP}_{j}(X) \otimes_{W, \iota} \mathbb{C}\right) .
$$


Moreover, by comparing the Hodge spectral sequence in Corollary 6.9 and the conjugate spectral sequence in Corollary 5.2 and using that the determinant is multiplicative on exact sequence, we furthermore conclude that

$$
\operatorname{det}\left(\mathrm{id}-q^{-s} \operatorname{Fr}_{q}^{*} \mid \operatorname{TP}_{j}(X) \otimes_{W, \iota} \mathbb{C}\right)=\left\{\begin{array}{l}
\operatorname{det}\left(\mathrm{id}-q^{-s} \operatorname{Fr}_{q}^{*} \mid H_{\text {crys }}^{\text {od }}(X / W) \otimes_{W, \iota} \mathbb{C}\right) \\
\operatorname{det}\left(\mathrm{id}-q^{-s} \operatorname{Fr}_{q}^{*} \mid H_{\text {crys }}^{\text {ev }}(X / W) \otimes_{W, \iota} \mathbb{C}\right)
\end{array}\right.
$$

respectively, as $j$ is odd or even. Putting the two equalities together, we find that

$$
\frac{\operatorname{det}_{\infty}\left(s \cdot \operatorname{id}-\Theta \mid \operatorname{TP}_{\text {od }}(X) \otimes_{W, \iota} \mathbb{C}\right)}{\operatorname{det}_{\infty}\left(s \cdot \operatorname{id}-\Theta \mid \operatorname{TP}_{\text {ev }}(X) \otimes_{W, \iota} \mathbb{C}\right)}=\frac{\operatorname{det}\left(\operatorname{id~}-q^{-s} \operatorname{Fr}_{q}^{*} \mid H_{\text {crys }}^{\text {od }}(X / W) \otimes_{W, \iota} \mathbb{C}\right)}{\operatorname{det}\left(\operatorname{id}-q^{-s} \operatorname{Fr}_{q}^{*} \mid H_{\text {crys }}^{\text {ev }}(X / W) \otimes_{W, \iota} \mathbb{C}\right)},
$$

and finally, it is proved in [2, Théorème VII.3.2.3] that the right-hand side is equal to the Hasse-Weil zeta function $\zeta(X, s)$.

By the analogue of analytic continuation, Proposition 6.6 the inverse Frobenius operator $\varphi^{-1}$ on $\mathrm{TF}_{*}(X ; p)$ gives rise to a meromorphic Frobenius operator

$$
\mathrm{TP}_{*}(X) \stackrel{\varphi}{\longrightarrow} \mathrm{TP}_{*}(X),
$$

which is defined and invertible after inverting $p$. We relate this operator to the geometric Frobenius $\mathrm{Fr}_{q}^{*}$ as follows. By functoriality, the Hodge spectral sequence

$$
E_{i, j}^{2}=\bigoplus_{m \in \mathbb{Z}} \lim _{n, F} H^{-i}\left(X, W_{n} \Omega_{X}^{j+2 m}\right) \Rightarrow \mathrm{TP}_{i+j}(X)
$$

is a spectral sequence of graded $\mathrm{TP}_{*}(k)$-modules. Moreover, on $E_{i, j}^{2}$, and hence, on $E_{i, j}^{\infty}$, the geometric Frobenius acts as

$$
\operatorname{Fr}_{q}^{*}=q^{w} \varphi^{r}
$$

where $w=j+m$ is the weight and $q=p^{r}$.

\section{Acknowledgements}

It is a great pleasure to thank the Hausdorff Research Institute for Mathematics for its hospitality and support during the Trimester Program "Homotopy theory, manifolds, and field theories," where part of the work reported here was conducted. I am also very grateful to Alain Connes for helpful conversations and for announcing Theorem $\mathrm{A}$ in his paper [13 and to Peter Scholze for explaining the conjugate spectral sequence to me and for making me fully appreciate the importance of Bökstedt's periodicity theorem.

\section{References}

[1] M. Artin, A. Grothendieck, and J. L. Verdier, Théorie des topos et cohomologie étale des schémas. Tome 2, Séminaire de Géometrie Algébrique du Bois-Marie 1963-1964 (SGA 4), Lecture Notes in Math., vol. 270, Springer-Verlag, New York, 1972.

[2] P. Berthelot, Cohomologie cristalline des schemas de caracteristique $p>0$, Lecture Notes in Math., vol. 407, Springer-Verlag, New York, 1974.

[3] B. Bhatt, M. Morrow, and P. Scholze, Integral p-adic Hodge theory and topological Hochschild homology, in preparation.

[4] A. J. Blumberg and M. A. Mandell, The strong Künneth theorem for topological periodic cyclic homology, arXiv:1706.06846

[5] L Localization theorems in topological Hochschild homology and topological cyclic homology, Geom. Topol. 16 (2012), 1053-1120.

[6] J. M. Boardman, Conditionally convergent spectral sequences, Homotopy invariant algebraic structures (Baltimore, MD, 1998), Contemp. Math., vol. 239, Amer. Math. Soc., Providence, RI, 1999, pp. 49-84. 
[7] M. Bökstedt, Topological Hochschild homology, Preprint, Bielefeld University, 1985.

[8] , Topological Hochschild homology of $\mathbb{Z}$ and $\mathbb{Z} / p$, Bielefeld, 1985.

[9] J. Borger, The basic geometry of Witt vectors, I. The affine case, Algebra Number Theory 5 (2011), 231-285.

[10] _ The basic geometry of Witt vectors, II. Spaces, Math. Ann. 351 (2011), 877-933.

[11] A. K. Bousfield, The localization of spectra with respect to homology, Topology 18 (1979), $257-281$

[12] H. Cartan, Séminaire 1954-1955. Algébre d'Eilenberg-Mac lane et homologie, Benjamin, New York, 1967.

[13] A. Connes, An essay on the Riemann Hypothesis, Open problems in mathematics, Springer, 2016, pp. 225-257.

[14] A. Connes and C. Consani, Cyclic homology, Serre's local factors and $\lambda$-operations, J. $K$ theory 14 (2014), 1-45.

[15] C. Deninger, Motivic L-functions and regularized determinants, Motives (Seattle, WA, 1991), Proc. Symp. Pure Math., vol. 55, Amer. Math. Soc., Providence, RI, 1994.

[16] O. Gabber, $K$-theory of henselian local rings and henselian pairs, Algebraic $K$-theory, commutative algebra, and algebraic geometry (Santa Margherita Ligure, 1989), Contemp. Math., vol. 126, Amer. Math. Soc., Providence, RI, 1992, pp. 59-70.

[17] T. Geisser and L. Hesselholt, Topological cyclic homology of schemes, K-theory (Seattle, 1997), Proc. Symp. Pure Math., vol. 67, 1999, pp. 41-87.

[18] T. Geisser and M. Levine, The K-theory of fields in characteristic p, Invent. Math. 139 (2000), 459-493.

[19] H. Gillet and R. Thomason, The K-theory of a strict hensel local ring and a theorem of Suslin, J. Pure Appl. Alg. 34 (1984), 241-254.

[20] J. P. C. Greenlees, Representing Tate cohomology of G-spaces, Proc. Edinburgh Math. Soc. (2) 30 (1987), 435-443.

[21] L. Hesselholt, On the p-typical curves in Quillen's K-theory, Acta Math. 177 (1997), 1-53.

[22] _ Topological Hochschild homology and the de Rham-Witt complex for $\mathbb{Z}_{(p)}$-algebras, Homotopy theory: Relations with algebraic geometry, group cohomology, and algebraic $K$ theory (Evanston, IL, 2002), Contemp. Math., vol. 346, Amer. Math. Soc., Providence, RI, 2004, pp. 253-259.

[23] $K$-theory of truncated polynomial algebras, Handbook of $K$-theory, Springer-Verlag, New York, 2005.

[24] On the topological cyclic homology of the algebraic closure of a local field, An Alpine Anthology of Homotopy Theory: Proceedings of the Second Arolla Conference on Algebraic Topology (Arolla, Switzerland, 2004), Contemp. Math., vol. 399, Amer. Math. Soc., Providence, RI, 2006, pp. 133-162.

[25] - The big de Rham-Witt complex, Acta Math. 214 (2015), 135-207.

[26] L. Hesselholt and I. Madsen, On the K-theory of finite algebras over Witt vectors of perfect fields, Topology 36 (1997), 29-102.

[27] - On the K-theory of local fields, Ann. of Math. 158 (2003), 1-113.

[28] On the de Rham-Witt complex in mixed characteristic, Ann. Sci. École Norm. Sup. 37 (2004), 1-43.

[29] L. Illusie, Complexe de de Rham-Witt et cohomologie cristalline, Ann. Scient. Éc. Norm. Sup. (4) 12 (1979), 501-661.

[30] L. Illusie and M. Raynaud, Les suites spectrales associées au complexe de de Rham-Witt, Inst. Hautes Études Sci. Publ. Math. 57 (1983), 73-212.

[31] J. F. Jardine, Local homotopy theory, Springer Monographs in Mathematics, Springer, New York, 2015.

[32] T. Nikolaus and P. Scholze, On topological cyclic homology, arXiv:1707.01799

[33] A. A. Suslin, On the K-theory of algebraically closed fields, Invent. Math. 73 (1983), 241-245.

[34] _ On the K-theory of local fields, J. Pure Appl. Alg. 34 (1984), 304-318.

[35] G. Tabuada, Finite generation of the numerical Grothendieck group, arXiv:1704.06252

[36] R. W. Thomason, Algebraic K-theory and étale cohomology, Ann. Scient. École Norm. Sup. 13 (1985), 437-552.

[37] S. Tsalidis, Topological Hochschild homology and the homotopy descent problem, Topology 37 (1998), 913-934. 
Nagoya University, Japan, and University of Copenhagen, Denmark

E-mail address: larsh@math.nagoya-u.ac.jp 